ThE ASTROPHYSICAL JouRnal, 347:727-742, 1989 December 15

(C) 1989. The American Astronomical Society. All rights reserved. Printed in U.S.A.

\title{
LONG-SLIT OPTICAL SPECTROSCOPY OF POWERFUL FAR-INFRARED GALAXIES: THE NATURE OF THE NUCLEAR ENERGY SOURCE
}

\author{
LeE ARMus ${ }^{1}$ \\ Astronomy Program, University of Maryland \\ TIMOTHY M. HeCKMAN ${ }^{1}$ \\ Department of Physics and Astronomy, The Johns Hopkins University \\ AND \\ GeORge K. Miley ${ }^{1}$ \\ Leiden Observatory \\ Received 1989 January 27; accepted 1989 June 17
}

\begin{abstract}
Optical spectroscopic data are presented for a sample of 47 powerful far-infrared galaxies (FIRGs) chosen with regard to IR spectral shape, and for six other IR-bright galaxies. The stellar absorption lines expected from a population of old stars (e.g., the Mg I " $b$ " band) are generally very weak in the nuclei $(<1 \mathrm{kpc})$ of these IR color-selected galaxies. In the cases for which we have adequate signal-to-noise ratio, we still find very weak $\mathrm{Mg}$ I absorption in regions well off the nucleus, implying that the visible spectrum is dominated by young stars and not by an AGN. At least one, and probably five, of the galaxies have detectable Wolf-Rayet emission features providing additional evidence for a young stellar population. About $20 \%$ of the galaxies have strong Balmer absorption lines, indicating the presence of a substantial intermediate-age $\left(10^{8}-10^{9} \mathrm{yr}\right)$ stellar population. The equivalent width of the $\mathrm{H} \alpha$ emission line can be modeled as arising from a mixture of a large young population, and an intermediate-age population of stars. Several pieces of evidence demonstrate that the spectra are usually observed through large column densities of cool gas and dust (steep Balmer decrements, strong Na D interstellar absorption lines, heavily reddened continua). The extinction-corrected optical continuum suggests that the young stellar population can typically provide at least $25 \%$ of the energy needed to power the far-infrared emission. We find that the emission-line spectra scatter between the loci of giant $\mathrm{H}$ II regions and LINERs in standard two-dimensional line ratio diagrams published by Baldwin, Phillips, and Terlevich in 1981. Examination of the spectrum of M82, the closest member of the sample, suggests that the primary mechanism for ionizing the emission-line gas changes from hot stars to shocks as the distance from the nucleus increases. This might explain the intermediate location of the more distant galaxies in the line ratio diagrams.
\end{abstract}

Subject headings: galaxies: nuclei — galaxies: stellar content - infrared: sources

\section{INTRODUCTION}

It has been known since the time of the IRAS mission that galaxies with far-infrared luminosities of $10^{11}-10^{12} L_{\odot}$ and with $L_{\text {FIR }} / L_{B}=10-100$ exist (Soifer et al. 1984). Through extensive modeling and observations of $\mathrm{H}$ il region/molecular cloud complexes in the Galaxy, the infrared radiation from these far-infrared galaxies (FIRGs) is believed to be primarily thermal emission from heated dust grains (see review of Stein and Soifer 1983). For the few powerful far-infrared galaxies studied in detail, it has been proposed that intense episodes of star formation provide the energy to heat the dust (Rieke et al. 1980, 1985; Gerhz, Sramek, and Weedman 1983; Cutri, Rieke, and Lebofsky 1984). While starburst models are consistent with the observational data over a large range in wavelengths, very little direct evidence exists for significant populations of young stars, and in many cases the presence of an active nucleus either cannot be ruled out, or is required on the basis of energy considerations. It is believed by some authors (Sanders et al. 1988) that the most powerful far-infrared galaxies may be dust-enshrouded quasars, in an early stage before

\footnotetext{
${ }^{1}$ Visiting Observer, Kitt Peak National Observatory, Cerro Tololo InterAmerican Observatory, National Optical Astronomy Observatory, operated by AURA, Inc. under contract with the National Science Foundation.
}

the nucleus is directly visible. Large-scale supernovae-driven winds are thought to operate in at least some of the galaxies (McCarthy, Heckman, and van Breugel 1987; Heckman, Armus, and Miley 1987), providing a means of eventually sweeping the circumnuclear regions clear of much of the obscuring dust and gas. It is true, however, that the relationship of powerful far-infrared galaxies to the accepted classes of active galaxies is still unclear.

In order to better understand the primary energy source responsible for heating the dust and ionizing the emission-line gas, we have undertaken a spectroscopic survey of galaxies from the IRAS catalog chosen to have far-infrared spectral energy distributions similar to the prototypical class members Arp 220, M82, NGC 6240, and NGC 3690. It was required that between $25 \mu \mathrm{m}$ and $60 \mu \mathrm{m}, \alpha \leq-1.5$, and that between $60 \mu \mathrm{m}$ and $100 \mu \mathrm{m}, \alpha \geq-0.5$, where $S_{v} \propto v^{\alpha}$. These IR criteria resulted in the generation of a sample of over 250 extragalactic objects, which were then identified using the Palomar Sky Survey and ESO/SRC Southern Sky Survey. Results from the optical nuclear spectra of 47 such galaxies are presented in this paper. It is obviously not a complete sample but rather one that will help us investigate the energy source behind the farinfrared, and nebular line emission seen in this type of galaxy. We also present results for six other infrared-bright galaxies. 
These galaxies will be included in the tables, but due to the size of the sample they will not be used in the statistical investigations to follow. For $\sim 40 \%$ of the infrared color-selected sample galaxies (ICSS galaxies), enhanced star formation alone can account for the nuclear spectral properties observed. A combination of young stars, an AGN, or shocks might operate in the circumnuclear regions of the remaining galaxies.

\section{OBSERVATIONS AND DATA REDUCTION}

Table 1 gives a list of the instruments used, their characteristics, and the dates upon which the nuclear spectra were obtained. In all cases the data have been flat-fielded, have had a HENEAR lamp-derived dispersion solution applied, and have been sky-subtracted using the standard procedures found in the NOAO's IRAF reduction package. From these twodimensional data, we have extracted a one-dimensional "nuclear" spectrum by summing over the innermost few arcseconds of the slit (see Table 1 for details). The 1985 December/ 1986 January and 1988 January data have been flux-calibrated in IRAF using KPNO IIDS standard stars.

Table 2 lists the 53 galaxies in order of right ascension, divided into two groups. The first group consists of 47 galaxies (53 nuclei) in our infrared color-selected sample (ICSS). Five of the galaxies have two nuclei located within the error bars at the IRAS position. One galaxy, NGC 3690 , has three nuclei at the IRAS position. We include data on all the nuclei in these cases, so that there are 53 ICSS nuclei in Table 2. The second group of six galaxies (denoted by asterisks) were chosen to be bright $\left(S_{v}>10 \mathrm{Jy}\right)$ at $60 \mu \mathrm{m}$. Along with the redshifts and infrared luminosities, the optical spectroscopic parameters are listed in the sixth through the thirteenth columns. Details concerning the values given in these columns can be found in the footnotes to the table. Throughout the paper, $H_{0}=75 \mathrm{~km} \mathrm{~s}^{-1} \mathrm{Mpc}^{-1}$ and $q_{0}=0$ are used.

\section{RESULTS}

\section{a) Emission-lines}

i) Line Ratios and Physical Conditions

Two-dimensional line flux ratio diagrams such as those studied by Baldwin, Phillips, and Terlevich (1981) are effective in segregating emission-line objects according to the dominant ionization mechanism. Figures $1 a$ and $1 b$ are two such diagrams in which we plot $\log [\mathrm{O}$ III $] / \mathrm{H} \beta$ versus $\log [\mathrm{N} \mathrm{II}] / \mathrm{H} \alpha$ and $\log \left[\mathrm{O}_{\mathrm{I}}\right] / \mathrm{H} \alpha$, respectively, for all the IR color-selected nuclei listed in Table 2 . Uncertainties in quoted line ratio values are generally \pm 0.02 in the log. The $\mathbf{H}$ II region models of McCall, Rybski, and Shields (1985) with values of the metallicity parameter $k \equiv(\mathrm{O} / \mathrm{H}) /(\mathrm{O} / \mathrm{H})_{\odot}$ ranging from 0.1 to 2.8 are also plotted, as is the line chosen by Veilleux and Osterbrock (1987) to empirically divide the domain of $\mathrm{H}$ II regions from that of active galactic nuclei (AGN). We also show the power-law $\left(f_{v} \propto v^{-1.5}\right)$ photoionization models of Ferland and Netzer (1983) for solar, and 1/10 solar abundances with varying values of the ionization parameter (the ratio of the density of ionizing radiation to gas density at the face of the emission-line clouds). Finally, the shock models of Shull and McKee (1979) for various shock speeds, preshock densities, metallicities, and ambient magnetic field are also indicated.

It is clear from Figures $1 a$ and $1 b$ that as a group, the galaxies in our sample do not have relative emission-line fluxes that can be comfortably assigned to either the AGN or the $\mathrm{H}$ II region class. In fact, the galaxies straddle the boundary between the $\mathrm{H}$ il regions (objects photoionized by hot stars) and the AGN (objects thought to be shock-heated or ionized by a nonthermal power-law continuum). Of the 53 ICSS nuclei in Table 2, 21 have spectra that are similar to $\mathrm{H}$ II regions, and 24 have spectra that are AGN-like (a nonstellar ionizing source indicated), based upon location in both line ratio diagrams. If an object falls to the right of the empirical dividing line described above (Veilleux and Osterbrock 1987) in Figure $1 a$ and Figure $1 b$, it is classified as AGN-like. If it falls to the left of the line, it is classified as $\mathrm{H}$ II like in all the discussions to follow. Four of the 53 nuclei have locations in these diagrams that move between the $\mathrm{H}$ II region and AGN domains from Figure $1 a$ to Figure $1 b$. The remaining four ICSS nuclei have too few detectable emission lines for classification. The ICSS FIRG nuclei without [O III $] / \mathrm{H} \beta$ listed in Table 2 are classified as $\mathrm{AGN}$-like if $\log [\mathrm{N} \mathrm{II}] / \mathrm{H} \alpha \geq-0.2$ and $\log [\mathrm{O} \mathrm{I}] /$ $\mathrm{H} \alpha \geq-1.2$, and they are classified as $\mathrm{H}$ II-like if $\log [\mathrm{N}$ II] $/$ $\mathrm{H} \alpha \leq-0.2$ and $\log \left[\mathrm{O}_{\mathrm{I}}\right] / \mathrm{H} \alpha \leq-1.2$. Nearly all the AGNlike galaxies have line ratios typical of LINERs: there are very few ICSS galaxies with the strong high-ionization lines typical of the narrow-line regions of Seyferts or quasars (two of the $13 \mathrm{AGN}$-like nuclei with measured $[\mathrm{O}$ III]/H $\beta$ have $[\mathrm{O} \mathrm{III}] / \mathrm{H} \beta>5$ ). We have no objects with the classical "broad line region" that is characteristic of type 1 Seyferts and quasars. As a result of the apparent dearth of "true" AGN among our IR color-selected galaxies, the reader should treat the terms AGN-like and LINER-like synonymously in the text and figures to follow.

Correcting the measured $\mathrm{H} \alpha$ and $\mathrm{H} \beta$ line fluxes for an

TABLE 1

OBSERVATIONS

\begin{tabular}{|c|c|c|c|c|c|}
\hline Date & Telescope & Instrument & Field of View ${ }^{\mathrm{a}}$ & $\begin{array}{c}\text { Spectral Range } \\
(\AA)\end{array}$ & $\begin{array}{c}\text { Resolution } \\
(\AA)\end{array}$ \\
\hline 1985 Dec ......... & CTIO $4 \mathrm{~m}$ & $\begin{array}{l}\text { Cassegrain Spectrometer and } \\
\text { GEC CCD }\end{array}$ & $1.5 \times 4 " .4$ & $5000-8500$ & 15.0 \\
\hline $1986 \mathrm{Feb} \ldots \ldots \ldots$ & KPNO $4 \mathrm{~m}$ & Cryogenic Camera & $3.2 \times 4.0$ & $4500-8000$ & 13.0 \\
\hline 1986 Nov .......... & KPNO $4 \mathrm{~m}$ & $\begin{array}{l}\text { Ritchey-Chrétien Spectrograph and } \\
\text { TI2 CCD }\end{array}$ & $2.0 \times 4.5$ & $6000-7000$ & 3.4 \\
\hline 1987 Jan $\ldots \ldots \ldots$ & CTIO $4 \mathrm{~m}$ & $\begin{array}{l}\text { Cassegrain Spectrometer and } \\
\text { GEC CCD }\end{array}$ & $2.0 \times 3.7$ & $6000-7000$ & 3.6 \\
\hline 1987 May ........ & KPNO $4 \mathrm{~m}$ & $\begin{array}{l}\text { Ritchey-Chrétien Spectrograph and } \\
\text { T12 CCD }\end{array}$ & $2.0 \times 4.5$ & $6000-7000$ & 3.6 \\
\hline 1988 Jan $\ldots \ldots \ldots$ & KPNO $4 \mathrm{~m}$ & $\begin{array}{l}\text { Ritchey-Chrétien Spectrograph and } \\
\text { T12 CCD }\end{array}$ & $2.0 \times 4.5$ & $4200-7000$ & 13.5 \\
\hline
\end{tabular}

a Extracted "nuclear" aperture taken from the long-slit spectrum. 
TABLE 2

Optical and Infrared Properties OF the ObSeRved Galaxies

\begin{tabular}{|c|c|c|c|c|c|c|c|c|c|c|c|c|}
\hline Galaxy & RA & DEC & $\mathrm{z}$ & $\mathrm{L}_{\mathrm{FIR}}$ & {$[\mathrm{OIII}] / \mathrm{H} \beta$} & {$[\mathrm{NII}] / \mathrm{H} \alpha$} & {$[\mathrm{SII}] / \mathrm{H} \alpha$} & {$[\mathrm{OI}] / \mathrm{H} \alpha$} & FWHM & $\mathrm{Mg}$ & $\mathrm{Na}$ & C65/C48 \\
\hline $00057+4021$ & 000545.0 & 402113 & 0.044 & 11.33 & & & & & & 3.5 & 4.0 & 1.45 \\
\hline $00182-7112$ & $0018 \quad 15.1$ & -711221 & 0.327 & 12.62 & 0.42 & -0.14 & -0.15 & -0.88 & 701 & $\leq 5.0$ & $\leq 3.0$ & \\
\hline $00188-0856$ & $\begin{array}{llll}0 & 18 & 53.3\end{array}$ & -085604 & 0.125 & 12.03 & & 0.10 & -0.35 & -0.92 & 387 & $\leq 3.0$ & $\leq 3.0$ & 1.40 \\
\hline $01003-2238$ & 010022.8 & -223809 & 0.118 & 11.84 & $0.54 \mathrm{~A}$ & $-0.70 \mathrm{~A}$ & $-0.53 \mathrm{~A}$ & $-0.95 \mathrm{~A}$ & $421 \mathrm{~B}$ & $\leq 1.0$ & $\leq 1.3$ & 0.67 \\
\hline $01173+1405$ & $\begin{array}{lll}01 & 1722.8\end{array}$ & 140554 & 0.031 & 11.37 & $\leq 0.01$ & -0.43 & -0.56 & -1.30 & & $\leq 0.5$ & 2.4 & \\
\hline $01217+0122$ & 012247.8 & 012255 & 0.138 & 12.01 & $1.18 \mathrm{C}$ & $-0.09 \mathrm{C}$ & $-1.12 \mathrm{C}$ & $-0.77 \mathrm{C}$ & 965D & $\leq 2.0$ & $\leq 2.0$ & 1.07 \\
\hline $01418+1651$ & 014148.1 & 165107 & 0.027 & 11.35 & & 0.10 & -0.23 & -0.67 & & 1.4 & 2.8 & 1.06 \\
\hline $02021-2104$ & 020206.3 & -210404 & 0.118 & 11.68 & & 0.08 & -0.02 & $\leq-0.70$ & & $\leq 1.5$ & 8.7 & 1.83 \\
\hline NGC 1222 & 030624.1 & -030848 & 0.009 & 10.39 & $\leq 0.48$ & -0.68 & -0.80 & -1.74 & 185 & $\leq 0.5$ & 1.7 & 0.82 \\
\hline $03514+1546$ & 035125.9 & 154654 & 0.021 & 10.81 & -0.54 & -0.32 & -0.73 & -1.47 & & 1.2 & 5.4 & 1.41 \\
\hline NGC 1614 & 043135.8 & -084057 & 0.016 & 11.27 & -0.12 & -0.22 & -0.78 & -1.83 & 297 & $\leq 0.4$ & 6.7 & 1.50 \\
\hline $05100-2425(\mathrm{a})$ & 051005.2 & -242529 & 0.034 & 11.04 & & 0.25 & -0.22 & -0.59 & 386 & 2.5 & 5.9 & 1.65 \\
\hline $05100-2425$ (b) & $\begin{array}{llll}05 & 10 & 05.2\end{array}$ & -242529 & 0.034 & 11.04 & 0.43 & -0.78 & -0.56 & $\leq-1.51$ & 62 & $\leq 2.0$ & $\leq 2.0$ & 1.01 \\
\hline $05447-2114$ & 054445.8 & -211444 & 0.040 & 10.73 & 0.20 & -0.24 & -0.48 & -1.26 & 290 & $\leq 1.0$ & 4.9 & \\
\hline $06361-6217$ & 063608.0 & -621753 & 0.160 & 12.03 & 0.17 & & & & & $\leq 2.5$ & $\leq 2.0$ & 1.09 \\
\hline $07205+7842$ & 072030.2 & 784242 & 0.087 & 11.40 & 0.60 & -0.15 & -0.53 & -1.01 & & 3.4 & 4.0 & 1.32 \\
\hline NGC 2623 & 083525.2 & 255549 & 0.019 & 11.27 & & -0.01 & -0.27 & -0.67 & 364 & 1.9 & 2.5 & 1.13 \\
\hline $08539-1208$ & 085359.1 & -120859 & 0.038 & 10.80 & 0.15 & -0.49 & -0.55 & -1.38 & 148 & $\leq 1.1$ & 2.5 & 1.14 \\
\hline $08572+3915$ (a) & $0857 \quad 13.0$ & 391539 & 0.058 & 11.71 & $\leq 0.25$ & -0.38 & & -1.13 & & $\leq 1.2$ & 1.9 & 0.92 \\
\hline $08572+3915$ (b) & 085713.0 & 391539 & 0.058 & 11.71 & $\leq 0.21$ & -0.51 & & & & $\leq 0.7$ & $\leq 0.4$ & 0.83 \\
\hline $09143+0939$ & $\begin{array}{lll}09 & 14 & 18.8\end{array}$ & 093923 & 0.047 & 11.26 & $\leq 0.08$ & -0.15 & -0.29 & -0.97 & 269 & $\leq 1.1$ & 2.7 & 1.09 \\
\hline $09168+0623$ & 091650.6 & 062341 & 0.083 & 11.40 & & -0.66 & -0.60 & -1.45 & & $\leq 2.0$ & $\leq 2.0$ & \\
\hline $09192+2124$ & $\begin{array}{lll}09 & 19 & 15.2\end{array}$ & 212402 & 0.078 & 11.59 & 0.25 & -0.40 & -0.55 & -1.21 & & $\leq 1.0$ & 3.0 & 1.35 \\
\hline M82 & 095142.4 & 695459 & 0.001 & 10.41 & -0.46 & -0.29 & -0.62 & -1.77 & 96 & $\leq 0.9$ & 5.0 & 1.56 \\
\hline NGC 3094 & 095841.9 & 160043 & 0.008 & 10.23 & & 0.01 & -0.31 & -0.86 & & 1.4 & 4.0 & 1.45 \\
\hline $10173+0828$ & 101722.0 & $\begin{array}{ll}08 & 2840\end{array}$ & 0.048 & 11.49 & & & & & & $\leq 1.0$ & 4.7 & 1.72 \\
\hline $10485-1447$ & 104834.6 & -144727 & 0.133 & 11.90 & & -0.16 & & -0.84 & 253 & $\leq 3.0$ & $\leq 3.0$ & 1.23 \\
\hline $10502-1843$ & 105016.5 & $-1843 \quad 12$ & 0.054 & 11.48 & & -0.19 & -0.41 & -1.06 & 309 & & 6.5 & \\
\hline $10565+2448$ & 105635.4 & 244843 & 0.042 & 11.71 & -0.22 & -0.34 & -0.61 & -1.32 & 210 & $\leq 0.8$ & 8.9 & 1.18 \\
\hline $11095-0238$ & 110930.2 & -023801 & 0.106 & 11.92 & 0.08 & -0.26 & -0.30 & -0.87 & 304 & $\leq 1.0$ & $\leq 1.0$ & 0.83 \\
\hline $11119+3257$ & 111157.4 & 325749 & 0.187 & 12.18 & 0.14 & $\leq-0.50$ & & $\leq-1.50$ & 1500 & $\leq 0.3$ & 9.2 & 1.95 \\
\hline $11120-2919$ & $\begin{array}{lll}11 & 12 & 05.4\end{array}$ & -291920 & 0.030 & 10.53 & & -0.03 & -0.40 & -1.00 & & $\leq 1.5$ & 3.3 & 1.37 \\
\hline $11122-2327$ & $\begin{array}{lll}11 & 12 & 14.4\end{array}$ & $-2327 \quad 19$ & 0.011 & 10.58 & & -0.31 & -0.57 & -1.59 & 252 & & & \\
\hline †NGC 3690(a) & 112542.1 & 585018 & 0.010 & 11.38 & 0.0 & -0.47 & -0.72 & -1.75 & & $\leq 0.3$ & 0.7 & 0.60 \\
\hline NGC 3690 (b) & 112542.1 & 585018 & 0.010 & 11.38 & -0.05 & -0.34 & -0.37 & -1.08 & 251 & $\leq 0.6$ & 1.5 & 1.14 \\
\hline NGC $3690(c)$ & 112542.1 & 585018 & 0.010 & 11.38 & & -0.34 & -0.50 & -1.24 & 311 & & & \\
\hline $11506-3851$ & 115040.2 & -385110 & 0.010 & 10.49 & & -0.24 & -0.57 & -1.42 & 310 & & & \\
\hline $12018+1941$ & 120151.8 & 194146 & 0.169 & 12.09 & $\geq 0.00$ & -0.04 & -0.26 & -0.49 & 426 & $\leq 1.0$ & 4.2 & 1.08 \\
\hline $12112+0305$ & $12 \quad 11 \quad 12.2$ & 030521 & 0.072 & 12.03 & 0.28 & -0.30 & -0.30 & -0.95 & & $\leq 1.0$ & 3.2 & 1.01 \\
\hline NGC 4194 & 121141.3 & $5448 \quad 10$ & 0.004 & 9.92 & $\leq 0.13$ & -0.34 & -0.65 & -1.44 & 204 & $\leq 0.4$ & 1.7 & 0.69 \\
\hline NGC 4418 & 122422.1 & -003614 & 0.007 & 10.65 & & $\leq 0.15$ & $\leq 0.03$ & $\leq-0.60$ & 155 & & & \\
\hline $13136+6223(\mathrm{a})$ & 131341.2 & 622318 & 0.033 & 11.38 & 0.04 & -0.38 & -0.67 & -1.49 & & $\leq 0.7$ & 1.3 & 0.91 \\
\hline $13136+6223(\mathrm{~b})$ & 131341.2 & $62 \quad 2318$ & 0.033 & 11.38 & $\leq 0.02$ & -0.37 & -0.52 & -1.32 & & 1.4 & 1.9 & 1.07 \\
\hline Mrk 273 & 134251.6 & $\begin{array}{lll}560814 & \end{array}$ & 0.037 & 11.86 & 0.64 & -0.06 & -0.29 & -0.90 & 519 & 0.8 & 4.0 & 1.05 \\
\hline $14348-1447$ & 143452.3 & -144725 & 0.081 & 12.03 & & $-0.25 \mathrm{E}$ & $-0.39 \mathrm{E}$ & $-1.26 \mathrm{E}$ & & & & \\
\hline $15225+2350$ & 152232.9 & 235036 & 0.138 & 11.84 & 0.22 & -0.32 & -0.61 & $\leq-1.25$ & 160 & $\leq 3.0$ & 3.3 & 1.29 \\
\hline $15250+3609$ & 152503.2 & 360902 & 0.053 & 11.66 & $0.79 \mathrm{E}$ & -0.32 & -0.39 & -1.04 & 373 & & & \\
\hline Arp 220 & 153246.3 & 234010 & 0.018 & 11.89 & 0.20 & 0.36 & -0.13 & -0.79 & 607 & & & \\
\hline NGC 6240 & 165027.7 & 022858 & 0.024 & 11.50 & 0.19 & 0.07 & -0.08 & -0.71 & 890 & & $7.9 \mathrm{~F}$ & \\
\hline $17132+5313(a)$ & $\begin{array}{lll}17 & 13 & 14.3\end{array}$ & 531351 & 0.051 & 11.60 & & -0.33 & -0.54 & -1.50 & 205 & & & \\
\hline $17132+5313(\mathrm{~b})$ & $\begin{array}{lll}17 & 13 & 14.3\end{array}$ & 531351 & 0.051 & 11.60 & & 0.14 & -0.07 & -0.60 & 182 & & & \\
\hline $22491-1808$ & 224909.4 & -180819 & 0.076 & 11.85 & $-0.01 E$ & $-0.34 \mathrm{E}$ & $-0.49 \mathrm{E}$ & $-1.62 \mathrm{E}$ & & & & \\
\hline $23488+2018$ & 234852.9 & 201820 & 0.018 & 11.11 & & -0.26 & -0.66 & -1.66 & 308 & & & \\
\hline *NGC 253 & 004507.8 & -253342 & 0.001 & 10.45 & & -0.11 & -0.40 & -1.36 & 274 & & & \\
\hline *NGC 660 & 014021.0 & 132318 & 0.003 & 10.09 & $\leq 0.46$ & -0.01 & -0.36 & -1.45 & 186 & 1.4 & 5.1 & 2.45 \\
\hline *NGC 2146 & 061045.0 & 782230 & 0.003 & 10.59 & -0.24 & -0.34 & -0.60 & -1.63 & & $\leq 1.0$ & 4.4 & 1.67 \\
\hline *NGC 3079 & 095834.8 & 555524 & 0.004 & 10.29 & $\geq 0.30 \mathrm{G}$ & $0.23 \mathrm{G}$ & $-0.10 \mathrm{G}$ & $-0.75 \mathrm{G}$ & $700 \mathrm{G}$ & 4.5 & 2.1 & 1.42 \\
\hline *NGC 3256 & 102542.0 & -433854 & 0.009 & 11.23 & & -0.21 & -0.66 & -1.66 & 209 & & & \\
\hline *Mrk 266(a) & $1336 \quad 15.0$ & 483154 & 0.028 & 11.17 & 0.15 & -0.22 & -0.40 & -0.95 & 414 & 1.7 & 2.1 & 1.00 \\
\hline *Mrk 266(b) & 133615.0 & 483154 & 0.028 & 11.17 & 0.60 & -0.26 & -0.45 & -1.35 & 388 & 1.3 & 1.8 & 1.19 \\
\hline
\end{tabular}

Col. (5).- The log of the far-infrared luminosity (in $L_{\odot}$ ) constructed using the far-infrared flux as defined in Cataloged Galaxies and Quasars Observed in the IRAS Survey (1985). For galaxies with multiple nuclei, the far-infrared luminosity for the entire system is listed for each nucleus.

Col. (6).-The log of the ratio of the flux of the [O III] $\lambda 5007$ emission line to that of $\mathrm{H} \beta$.

Col. (7).-The log of the ratio of the flux of the $[\mathrm{N} \mathrm{II}] \lambda 6583$ emission line to that of $\mathrm{H} \alpha$.

Col. (8).-The log of the ratio of the flux of the sum of the [S $\mathrm{SI}] \lambda 6717,6731$ emission lines to that of $\mathrm{H} \alpha$.

Col. (9).-The log of the ratio of the flux of the [O I $] \lambda 6300$ emission line to that of $\mathrm{H} \alpha$.

Col. (10).- The FWHM (in $\mathrm{km} \mathrm{s}^{-1}$ ) of $\mathrm{H} \alpha$, corrected for the instrumental profile.

Col. (11). - The equivalent width (in $\AA$ ) of the $\mathrm{Mg}$ I $\lambda 5174$ absorption line.

Col. (12).- The equivalent width (in $\AA$ ) of the Na I D $\lambda \lambda 5890,5896$ absorption lines.

Col. (13). - The ratio of the flux density $\left(F_{\lambda}\right)$ in the continuum measured at $6500 \AA$ to that measured at $4800 \AA$.

Notes.- + : The nucleus labeled (a) corresponds to nucleus C from Gehrz et al. 1983; the nucleus labeled (b) corresponds to nucleus A from Gehrz et al. 1983; and the nucleus labeled (c) corresponds to nucleus B from Gehrz et al. 1983. *: These galaxies were selected by IR flux ( $\left.S_{60 \mu \mathrm{m}}>10 \mathrm{Jy}\right)$, and not IR color (see text). A: Line ratios here are for the narrow components of the emission lines; B: FWHM of $\mathrm{H} \alpha$ is from best Gaussian fit to $\mathrm{H} \alpha$, [N $\mathrm{NI}]$ blend; C: Line ratios here are from best Gaussian fits to emission lines; D: The FWHM listed here is actually for the [O I] $\lambda 6300$ emission line; E: Data for this ICSS FIRG is from Sanders et al. 1988; F: Data for this ICSS FIRG is from Fosbury and Wall 1979; G: Data from Heckman, Balick, and Crane 1980. 


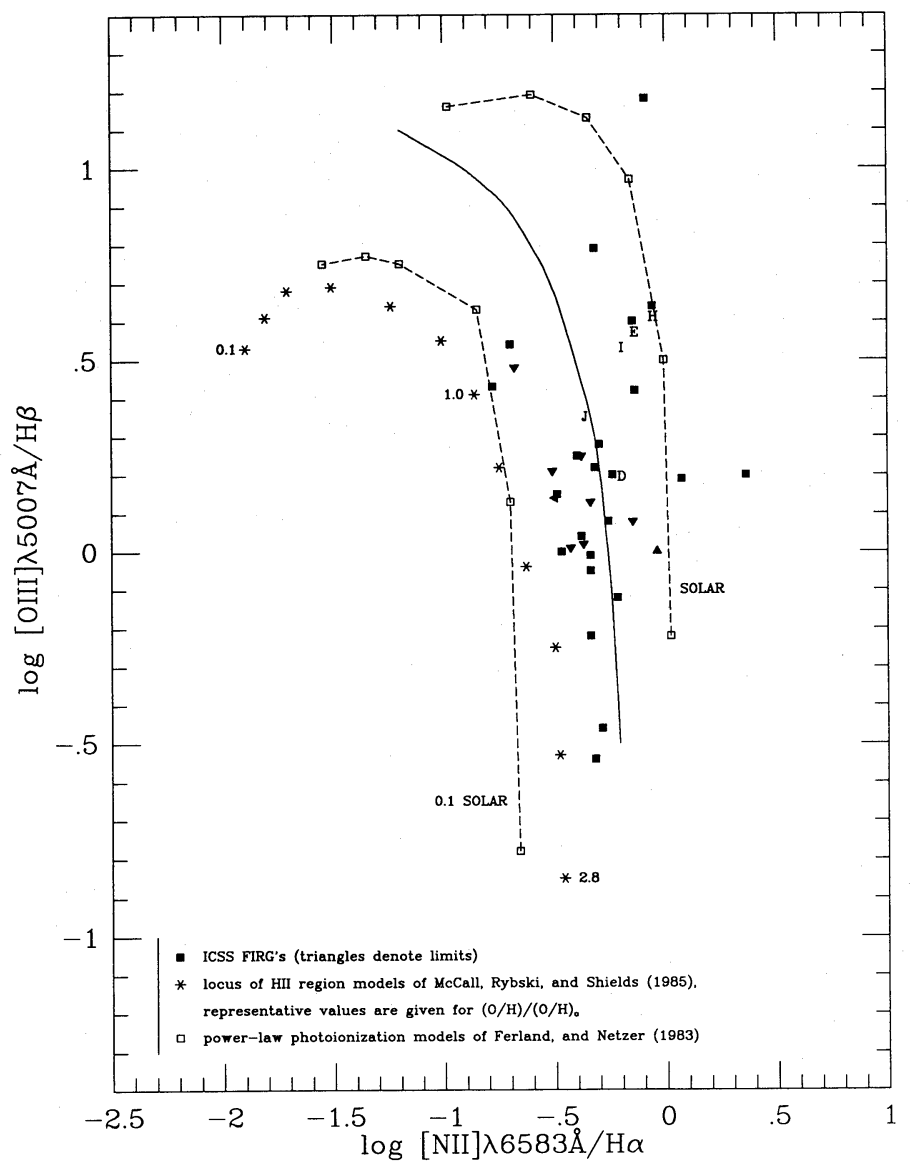

Fig. $1 a$

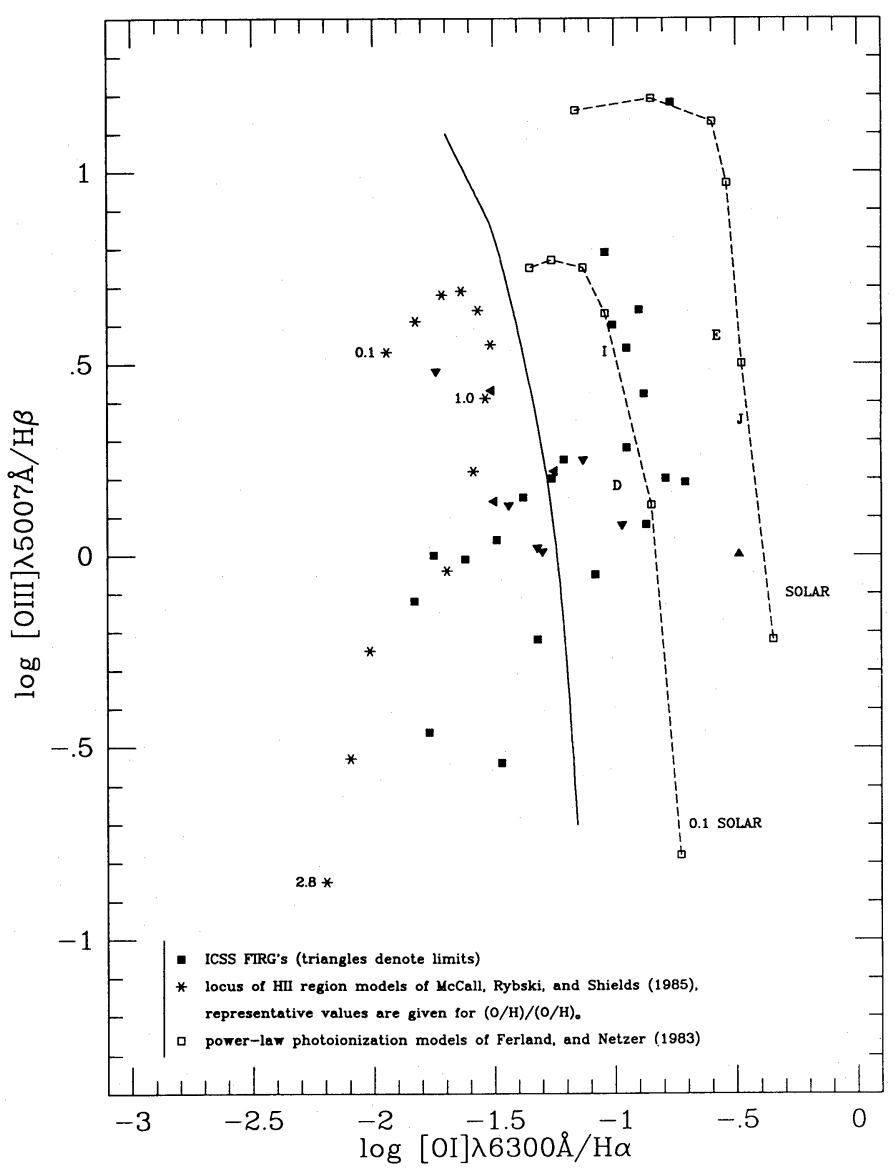

Fig. $1 b$

FIG. 1.- (a) Diagnostic emission-line flux ratio diagram for the infrared color-selected FIRGs. Triangles denote limits. The solid line taken from Veilleux and Osterbrock (1987) divides the empirical domains of AGNs from that of $\mathrm{H}$ II regions. Capital letters denote the positions in the diagram of the shock models of Shull and McKee (1979). The dashed lines indicate the power-law $\left(f_{v} \propto v^{-1.5}\right)$ photoionization models of Ferland and Netzer (1983), for solar, and one-tenth solar, abundances. For each of these curves, the ionization parameter $(U)$ varies from $10^{-4}$ to $10^{-1.5}$. Stars indicate the locations of the $\mathrm{H}$ II region models of McCall, Rybski, and Shields (1985). (b) Same as Fig. $1 a$, but for $[\mathrm{O} \mathrm{III}] / \mathrm{H} \beta$ vs. [O I $] / \mathrm{H} \alpha$.

underlying stellar absorption of $3 \AA$ equivalent width (see below) will lower all the diagnostic line ratios. None of the galaxies with $\mathrm{EQW}_{\mathrm{H} \alpha}$ or $\mathrm{EQW}_{\mathrm{H} \beta} \leq 10 \AA$ (these galaxies would have their line ratios lowered by $\geq 33 \%$ ) would need to be reclassified due to this correction. The galaxy NGC 4418 (affected the most of all the galaxies in this category), would have its $\log [\mathrm{N} \mathrm{II}] / \mathrm{H} \alpha$ lowered to $\sim-0.25$, but its $\log \left[\mathrm{O}_{\mathrm{I}}\right] / \mathrm{H} \alpha$ lowered to only $\sim-1.00$. Since both ratios do not change enough to require NGC 4418 to be reclassified as $\mathrm{H}$ II-like it will remain a member of the AGN-like group of ICSS FIRGs in the discussions to follow.

Three galaxies $(01418+1651$, NGC 2623 , and NGC 3094) have Balmer absorption lines that dominate the emission lines in the blue part of their spectra. For these ICSS FIRGs the equivalent width of the $\mathrm{H} \beta$ absorption line can be measured directly. We measure $6.5 \AA, 5.2 \AA$, and $1.2 \AA$, respectively. When these equivalent widths are used to correct the $\mathrm{H} \alpha$ emission, the red line ratios move closer to the dividing line (see above), but the classifications of these galaxies remain unchanged.

The highly anomalous object IRAS $11119+3257$ will be the subject of a future paper. It is characterized by extremely broad lines (see Table 2) and strong $\mathrm{Fe}$ II multiplets of the type seen only in quasars and type 1 Seyfert nuclei. The only detectable forbidden lines are the [O III] $\lambda 4363,4959$, and 5007 transitions, and these are as broad as the Balmer lines. It is starlike in a deep KPNO $4 \mathrm{~m} \mathrm{CCD} \mathrm{image.} \mathrm{While} \mathrm{it} \mathrm{falls} \mathrm{into} \mathrm{the} \mathrm{regime}$ of $\mathrm{H}$ II regions in the emission-line flux ratio diagrams, it is clearly a highly peculiar quasar. We will label IRAS $11119+3257$ in the figures and will classify it as having an "AGN-like" nuclear spectrum for the purposes of the statistical investigations to follow.

Comparison with the shock models of Shull and McKee (1979) shows that model $\mathrm{D}$, with a shock speed of $90 \mathrm{~km} \mathrm{~s}^{-1}$, most closely reproduces the relative emission-line strengths seen in the AGN-like galaxies. The power-law photoionization models of Ferland and Netzer (1983) do not have line ratios simultaneously consistent with both the $[\mathrm{N} \mathrm{II]} / \mathrm{H} \alpha$ and the $[\mathrm{O} \mathrm{I}] / \mathrm{H} \alpha$ observed in our sample galaxies.

Table 3 lists the electron densities in the emission-line nebulae. The densities were derived using the ratio of the [S II] 226717, 6731 lines, and the atomic parameters in Canto et al. (1980). The mean $\log n \sim 2.54 \pm 0.06$, for the ICSS FIRGs, and $\sim 2.68 \pm 0.07$ for the seven nuclei in Table 3 not selected for their far-infrared colors (denoted by asterisks).

For the nine nuclei with the strongest emission lines (largest fluxes and equivalent widths) we have measured electron temperatures using the reddening-corrected flux ratios of the 
TABLE 3

ELECTRON Densities

\begin{tabular}{|c|c|c|}
\hline $\begin{array}{c}\text { Galaxy } \\
\text { (1) }\end{array}$ & $\begin{array}{c}R \\
(2)\end{array}$ & $\begin{array}{c}\text { Density } \\
\text { (3) }\end{array}$ \\
\hline $01003-2238$ & 1.51 & $<2.00$ \\
\hline $01173+1405$ & 1.32 & 2.14 \\
\hline NGC $1222 \ldots$ & 1.12 & 2.66 \\
\hline NGC $1614 \ldots \ldots \ldots \ldots . .$. & 0.87 & 3.08 \\
\hline $05100-2425(a)$ & 1.14 & 2.62 \\
\hline $05100-2425(b) \ldots \ldots \ldots \ldots$ & 1.29 & 2.24 \\
\hline $05447-2114 \ldots \ldots \ldots \ldots$ & 1.16 & 2.58 \\
\hline $06361-6217 \ldots \ldots \ldots \ldots$ & 1.19 & 2.52 \\
\hline $08539-1208 \ldots$ & 1.32 & 2.14 \\
\hline $09143+0939$. & 1.10 & 2.70 \\
\hline $09168+0623 \ldots \ldots \ldots \ldots \ldots$ & 1.23 & 2.42 \\
\hline M82 ........... & 0.94 & 2.96 \\
\hline NGC 3094 & 1.20 & 2.50 \\
\hline $10502-1843$ & 1.13 & 2.64 \\
\hline $10565+2448 \ldots \ldots \ldots \ldots \ldots$ & 1.03 & 2.84 \\
\hline $11095-0238 \ldots \ldots \ldots \ldots$ & 1.28 & 3.28 \\
\hline $11120-2919 \ldots$ & 0.98 & 2.90 \\
\hline $11122-2327$ & 1.23 & 2.42 \\
\hline NGC $3690(a) \ldots \ldots \ldots \ldots$ & 1.16 & 2.58 \\
\hline NGC $3690(b) \ldots$ & 1.36 & 1.96 \\
\hline NGC $3690(c) \ldots \ldots \ldots \ldots$ & 1.21 & 2.48 \\
\hline $11506-3851 \ldots \ldots \ldots \ldots$ & 1.13 & 2.64 \\
\hline $12112+0305 \ldots \ldots \ldots \ldots \ldots$ & 1.91 & $<2.00$ \\
\hline NGC $4194 \ldots \ldots$. & 1.02 & 2.85 \\
\hline NGC 4418 & 1.11 & 2.68 \\
\hline $13136+6223(a) \ldots$ & 0.92 & 3.00 \\
\hline $13136+6223(b) .$. & 1.07 & 2.74 \\
\hline Mrk $273 \ldots \ldots \ldots \ldots \ldots$ & 1.16 & 2.58 \\
\hline $15250+3609 \ldots$ & 1.27 & 2.30 \\
\hline Arp $220 \ldots \ldots \ldots \ldots \ldots$ & 1.44 & $<2.00$ \\
\hline $17132+5313(\mathrm{a})$ & 1.26 & 2.36 \\
\hline $17132+5313(b) \ldots \ldots \ldots \ldots$ & 1.22 & 2.46 \\
\hline $23488+2018 \ldots \ldots \ldots \ldots$ & 1.04 & 2.80 \\
\hline *NGC $253 \ldots$ & 1.07 & 2.74 \\
\hline *NGC 660 & 1.17 & 2.57 \\
\hline${ }^{*}$ NGC $2146 \ldots \ldots \ldots \ldots$ & 1.25 & 2.40 \\
\hline${ }^{*}$ NGC 3079 ............. & 1.11 & 2.68 \\
\hline *NGC 3256 & 0.94 & 2.96 \\
\hline *Mrk 266(a) & 0.96 & 2.94 \\
\hline${ }^{*}$ Mrk 266(b) ............ & 1.20 & 2.50 \\
\hline
\end{tabular}

Col. (2).-The ratio of the [S II] $\lambda 6717$ emission line flux, to the [S II] $\lambda 6731$ emission-line flux.

Col. (3).-The log of the electron density (in $\mathrm{cm}^{-3}$ ) calculated for the emission-line nebulae, as derived from the [S II] ratio of col. (2).

NoTE.-Galaxies marked with an asterisk $\left({ }^{*}\right)$ were selected by IR flux ( $\left.S_{60 \mu \mathrm{m}}>10 \mathrm{Jy}\right)$, and not IR color (see text).

[O III] $\lambda \lambda 4959,5007 /[\mathrm{O}$ III $] \lambda 4363$ and the $[\mathrm{N}$ II $] \lambda \lambda 6548,6583 /$ $[\mathrm{N}$ II] $\lambda 5755$ lines (cf. Osterbrock 1988). The $4363 \AA$ and 5755 $\AA$ lines are very weak and are sometimes on top of, or near, weak stellar absorption lines. Thus, the temperatures we measure are uncertain by typically $0.1-0.2$ dex. For the nine nuclei [M82, NGC 1222, NGC 1614, NGC 3690(a), NGC 4194, Mrk 266(a) and (b), Mrk 273, and IRAS 13136+6223(a)] we find that the [O III] ratio implies typically $T=14,000-22$, $000 \mathrm{~K}$, while the $[\mathrm{N} \mathrm{II}]$ lines typically imply $T=8000-13,000$ $\mathrm{K}$. Models of low-density gas photoionized by hot stars, by an AGN, and collisionally ionized by a shock predict $[\mathrm{O}$ III $]$ temperatures of $4000-14,000 \mathrm{~K}, 9000-18,000 \mathrm{~K}$, and $25,000-30,000$ K, respectively (McCall, Rybski, and Shields 1985; Ferland and Netzer 1983; Kraemer 1985; Binette, Dopita, and Tuohy $1985)$. For the $[\mathrm{N} \mathrm{II}]$ temperatures, the corresponding values are $4500-12,000 \mathrm{~K}, \sim 10,000 \mathrm{~K}$, and $7000-8500 \mathrm{~K}$. The [O III] temperatures, therefore, weakly favor photoionization as the dominant energy source in most of the nuclei.

In order to take advantage of the spatial resolution attainable for the nearest of our galaxies, the emission-line properties as a function of nuclear distance were studied in M82 (see also McCarthy, Heckman, and van Breugel 1987). A position angle of $151^{\circ}$, which lies along the minor axis, was chosen for this study. While the $[\mathrm{N} \mathrm{II]} / \mathrm{H} \alpha$, and $[\mathrm{S} \mathrm{II}] / \mathrm{H} \alpha$ ratios show no overall radial trend, the $\left[\mathrm{O}_{\mathrm{I}}\right] / \mathrm{H} \alpha$ ratio changes by about a factor of 20 (from 0.01 in the nucleus, to 0.20 at $r \geq 1 \mathrm{kpc}$ ). Over the same range in radius, the $[\mathrm{O} \mathrm{III}] / \mathrm{H} \beta$ ratio rises from $\sim 0.3$ in the nucleus, to $\sim 1.0$ for $r \geq 1 \mathrm{kpc}$. This progression is illustrated in the diagnostic line ratio diagram of Figure 2, where it is clear that the nucleus of M82 is a member of the $\mathrm{H}$ II region class, while the off-nuclear spectrum is that of a LINER. It is possible that many of the galaxies in our sample would exhibit similar behavior if they could be examined with comparable resolution. A full discussion of the off-nuclear properties of all of the galaxies in our sample will be presented in a future paper.

ii) Line Widths and Shapes

The average value of H $\alpha$ FWHM for the ICSS FIRG nuclei listed in Table 2 is $372 \mathrm{~km} \mathrm{~s}^{-1}$, with a standard deviation in the mean of $287 \mathrm{~km} \mathrm{~s}^{-1}$. The H $\alpha$ FWHM is given only for the 33 nuclei with high-resolution spectra. Uncertainties in the

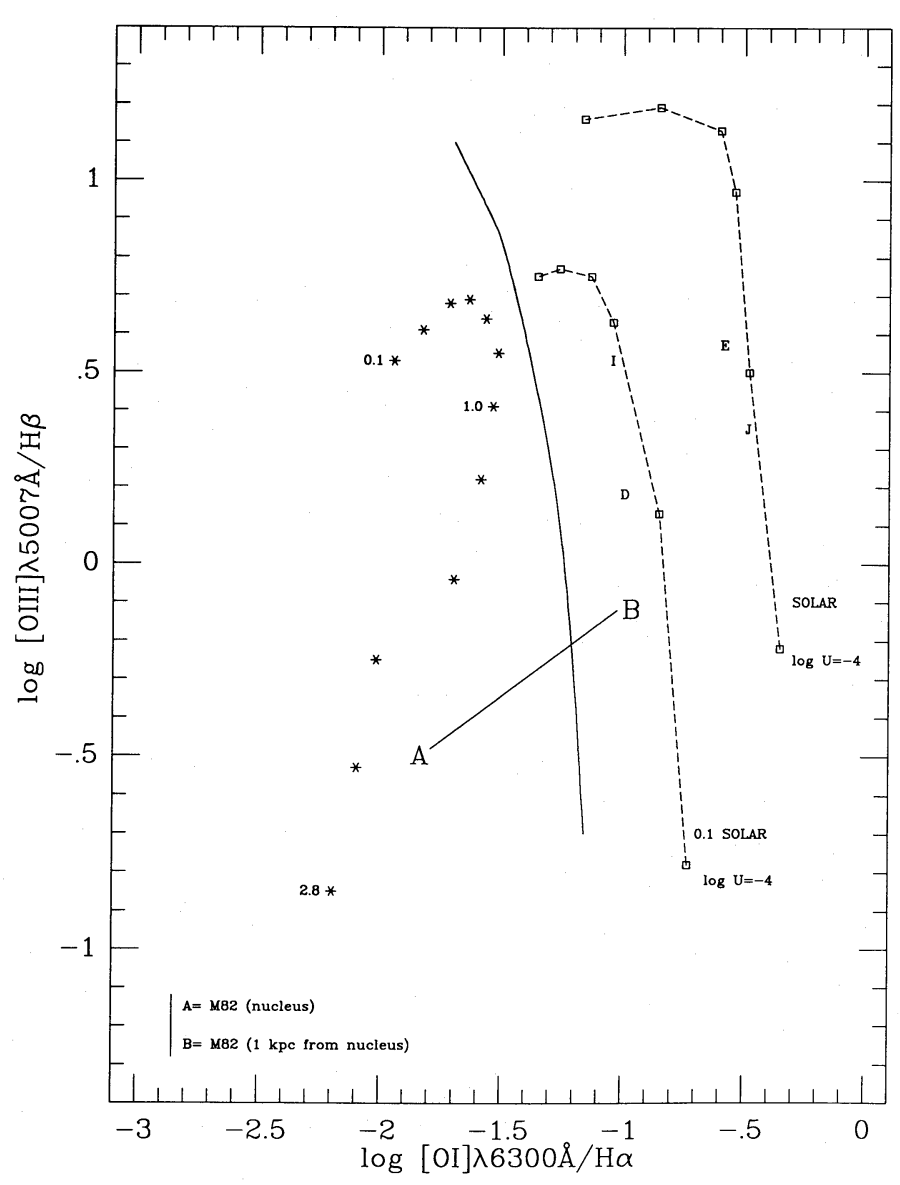

FIG. 2.-Same as in Fig. $1 b$, but including a vector representing the change in the emission-line flux ratios in M82, as gas is sampled at up to $1 \mathrm{kpc}$ from the nucleus along a P.A. $=151^{\circ}$. 

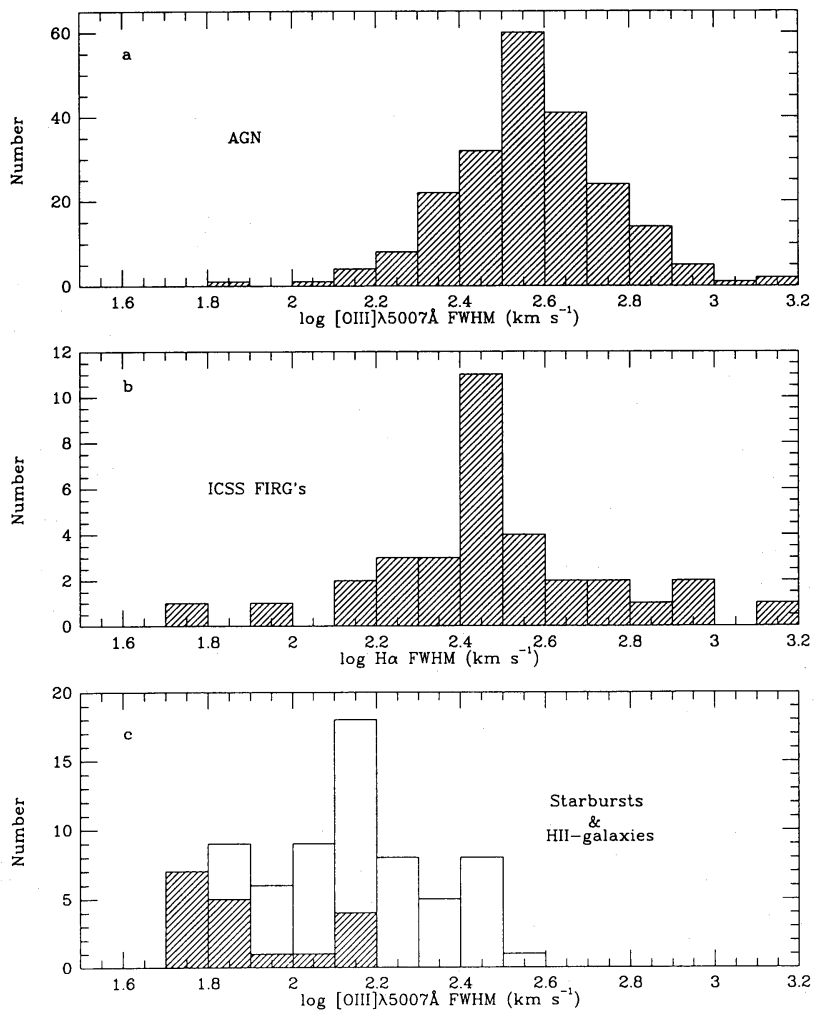

FIG. 3. $-(a)$ Distribution of the [O III] $\lambda 5007$ FWHM for the AGN of Wilson and Heckman (1984), combined with the LINERs of Whittle (1985); $(b)$ the H $\alpha$ FWHM for the ICSS FIRGs; and (c) the [O III] $\lambda 5007$ FWHM for the starburst galaxies (open) and $\mathrm{H}$ il galaxies (shaded) of Whittle (1985).

quoted values for the $\mathrm{H} \alpha \mathrm{FWHM}$ are typically $10 \%$. Of the ICSS nuclei with $\mathrm{H} \alpha$ FWHM, 11 (33\%) have $\mathrm{H} \alpha$ $F W H M \geq 350 \mathrm{~km} \mathrm{~s}^{-1}$. These "broad-line objects" have similar forbidden line widths: galaxies with very wide permitted lines, and much narrower forbidden lines (as in Seyfert 1 nuclei, or quasars) are not seen. Figure 3 compares the $\mathrm{H} \alpha$ line widths of the spectrally resolved ICSS FIRGs to the [O III] linewidths of various classes of active galactic nuclei taken from the literature (Whittle 1985; Wilson and Heckman 1984). The median of the distribution for the ICSS FIRG nuclei is about $30 \%$ lower than it is for the AGN, and about twice the median for the starbursts. This implies that the emission lines of our IR color-selected galaxies have widths that are, on average, intermediate between those found in the narrow-line region (NLR) of $A G N$, and those found in starburst galaxies. It is also clear however, that there is a large spread in the $\mathrm{H} \alpha$ FWHM (62-1500 $\left.\mathrm{km} \mathrm{s}^{-1}\right)$ for the ICSS FIRG nuclei, and therefore line width alone is not a sufficient parameter to describe this class of galaxy. Since the $\mathrm{H} \alpha \mathrm{FWHM}$ in the nuclei of our sample galaxies matches the FWHM of the other emission lines (e.g., [O I] $\lambda 6300,[\mathrm{~N}$ II $] \lambda \lambda 6548,6584)$ by better than $20 \%$, we feel the use of $\mathrm{H} \alpha$ to characterize a general line width in these galaxies is justified. In Figure 4 we see that the ICSS FIRGs classified as AGN-like, based upon their nuclear emission-line flux ratios (see above), have wider lines, on average, than do the ICSS FIRGs with $\mathrm{H}$ II region-like nuclei (493 $\mathrm{km} \mathrm{s}^{-1}$ vs. $211 \mathrm{~km} \mathrm{~s}^{-1}$, respectively). The median of the distribution for the AGN-like ICSS FIRGs is the same, within the measurement uncertainties, as the median of the distribution for the "true" AGN of Figure 3. The median of the dis- tribution for the $\mathrm{H}$ II-like ICSS FIRGs however, is still about 1.6 times that found for the starburst galaxies of Figure 3. Thus while the emission-line flux ratios of these FIRGs indicate that no other energy source besides hot stars is required to ionize the gas, the line widths are almost twice those found in galaxies thought to be undergoing episodes of enhanced star formation (starbursts).

Of the 33 ICSS FIRG nuclei with high resolution spectra, 11 $(33 \%)$ have composite line profiles which consist of relatively narrow line cores, plus broad, blueshifted asymmetrical bases. In both the AGN-like and the $\mathrm{H}$ II-like groups, $\sim 30 \%$ of the nuclei with high spectral resolution have blue wings on their emission-line profiles. Examples of the nuclear spectra of two of the galaxies having emission-line profiles with blue wings are presented in Figure 5. We note that Mirabel and Sanders (1988) find that the optical emission-line redshifts of FIRGs are systematically blueshifted by $\sim 100 \mathrm{~km} \mathrm{~s}^{-1}$ on average with respect to the $\mathrm{H}_{\mathrm{I}} 21 \mathrm{~cm}$ redshifts (presumably a good measure of the systemic velocity). This blueshift is probably related to the blue asymmetries in profile shape noted above. Both phenomenon suggest that radial flow plus dust obscuration are influencing the optical emission-line profiles.

iii) Balmer Line Luminosities and Equivalent Widths

Table 4 lists the observed equivalent widths of $\mathrm{H} \alpha$ and $\mathrm{H} \beta$, the observed Balmer decrement, the observed flux of $H \alpha$, and the reddening-corrected luminosity of $H \alpha$. The observed flux was corrected for reddening using the $\mathrm{H} \alpha / \mathrm{H} \beta$ ratio of the fourth column, assuming case $\mathrm{B}$ recombination and a standard interstellar extinction curve (Osterbrock 1988). For the ICSS FIRG nuclei $F_{\mathrm{H} \alpha}\left(\right.$ in ergs $\mathrm{cm}^{-2} \mathrm{~s}^{-1}$ ) ranges from a low of
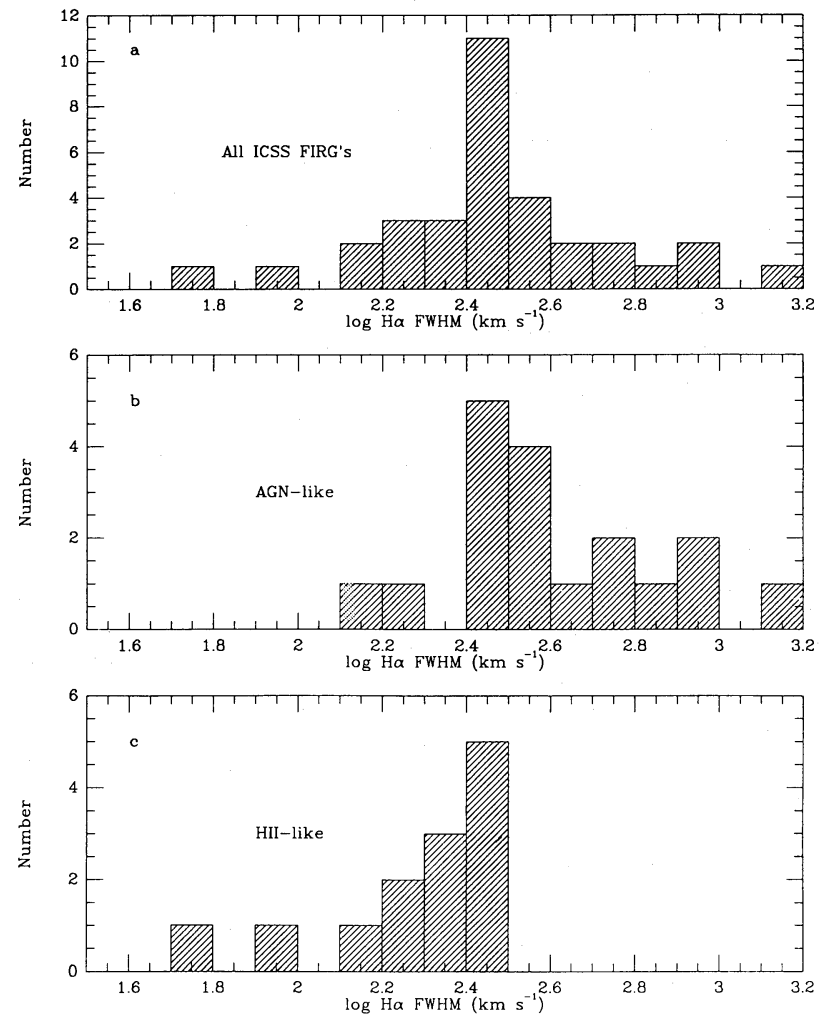

FIG. 4.- (a) Distribution of the H $\alpha$ FWHM for all the ICSS FIRGs, $(b)$ for the ICSS FIRGs with nuclear spectra resembling AGN (LINERs), and (c) for the ICSS FIRGs with nuclear spectra resembling $\mathrm{H}$ II regions. 

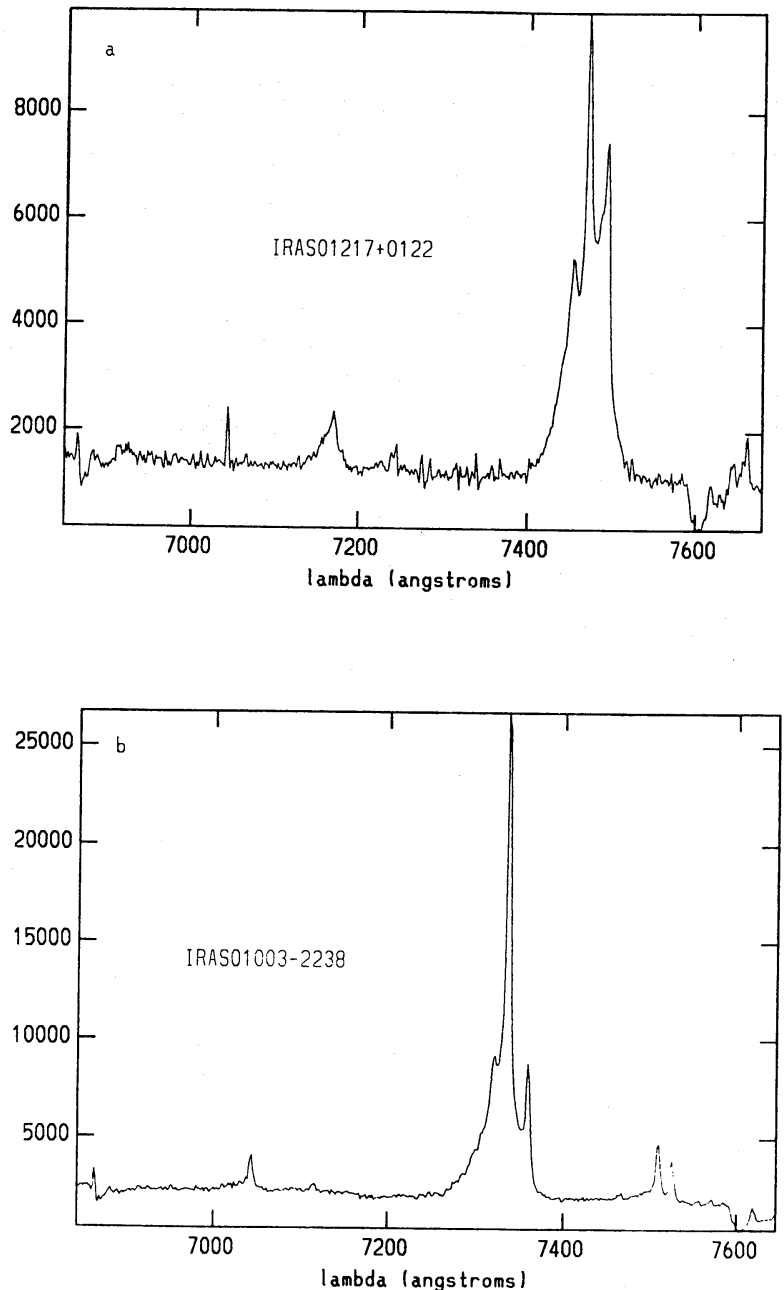

FIG. 5.-(a) The nuclear spectrum of IRAS $01217+0122$ (vertical scale is in relative flux units), and (b) the nuclear spectrum of IRAS 01003 - 2238 (vertical scale is in relative flux units), both showing the broad, blue asymmetric emission-line profiles. The strongest feature in both spectra is the $\mathrm{H} \alpha,[\mathrm{N} \mathrm{II}]$ blend.

$\sim 1.8 \times 10^{-15}$ in IRAS $12018+1941$, to a high of $\sim 6.8 \times 10^{-13}$ in M82. Also $L_{\mathrm{H} \alpha}$ (in ergs $\mathrm{s}^{-1}$ ) ranges from $\sim 1.6 \times 10^{40}$ in M82, to the highest measured value of $\sim 4.3 \times 10^{43}$ in IRAS $11119+3257$. We can compare the $\mathrm{H} \alpha$ emission-line properties of our IR color-selected FIRGs, to a sample of $\mathbf{H}$ II region nuclei, Markarian starburst nuclei, and disk H II regions from Kennicutt, Keel, and Blaha (1989). The $\mathrm{H}$ II region nuclei are from a sample of normal S-Irr galaxies chosen to have $\log \left[\mathrm{N}_{\mathrm{II}}\right] / \mathrm{H} \alpha \leq-0.15$, and the starburst nuclei (required to have $\log [\mathrm{N} \mathrm{II}] / \mathrm{H} \alpha \leq-0.15$ also) are taken from Balzano (1983). The median value of $\log L_{\mathrm{H} \alpha}$ is $\sim 39.5$ for the nuclear $\mathbf{H}$ II regions, $\sim 39.3$ for the brightest disk $H$ II regions, and $\sim 40.7$ for the Markarian starburst nuclei. The three distributions have not been corrected for reddening. Without applying a reddening correction to our ICSS FIRG nuclei, the median $\log L_{\mathrm{H} \alpha}$ is $\sim 41.0$. Our IR color-selected nuclei then, exhibit $\mathrm{H} \alpha$ luminosities that are most similar to Markarian starburst nuclei, and much higher than that found for bright disk or nuclear $H$ II regions. Since the median Balmer decrement in the ICSS FIRG nuclei is larger than that
TABLE 4

BALMER LiNe LuMinosities AND EQUiVALENT WIDTHS

\begin{tabular}{|c|c|c|c|c|c|}
\hline Galaxy & $\mathrm{EQW}_{\mathrm{H} \beta}$ & $\mathrm{EQW}_{\mathrm{H} \alpha}$ & $\mathrm{H} \alpha / \mathrm{H} \beta$ & $\mathrm{F}_{\mathrm{H} \alpha}$ & $\mathrm{L}_{\mathrm{H} \alpha}$ \\
\hline \multicolumn{6}{|l|}{$00057+4021$} \\
\hline $00182-7112$ & 14.4 & 102.6 & & & \\
\hline 00188-0856 & & 22.0 & & -14.60 & \\
\hline $01003-2238$ & 12.2 & 112.0 & 6.16 & -14.07 & \multirow{2}{*}{42.11} \\
\hline $\begin{array}{l}01173+1405 \\
01217+0122\end{array}$ & $\begin{array}{l}19.0 \\
11.3\end{array}$ & $\begin{array}{l}116.2 \\
1002\end{array}$ & \multirow{3}{*}{9.46} & & \\
\hline $01418+1651$ & & 7.5 & & -14.17 & \\
\hline $02021-2104$ & & 8.7 & & -14.00 & \\
\hline NGC 1222 & 17.1 & 127.2 & 6.90 & -12.39 & 41.61 \\
\hline $03514+1546$ & 9.0 & 58.9 & 10.08 & -13.46 & 41.63 \\
\hline $\begin{array}{c}\text { NGC } 1614 \\
05100-2425(a)\end{array}$ & 31.8 & $\begin{array}{r}175.7 \\
5.0\end{array}$ & 8.67 & -12.28 & 42.44 \\
\hline $05100-2425$ (b) & 8.3 & 49.0 & \multirow[t]{2}{*}{4.12} & -14.24 & \multirow[t]{2}{*}{40.45} \\
\hline $\begin{array}{l}05447-2114 \\
06361-6217\end{array}$ & 3.1 & 44.1 & & -13.50 & \\
\hline $\begin{array}{r}06361-6217 \\
07205+7842\end{array}$ & $\begin{array}{r}13.4 \\
8.6\end{array}$ & 46.0 & \multirow{2}{*}{7.08} & -13.64 & \multirow{2}{*}{42.39} \\
\hline NGC 2623 & & 13.3 & & $\begin{array}{l}-13.04 \\
-14.24\end{array}$ & \\
\hline 08539-1208 & 15.3 & 126.2 & \multirow{4}{*}{$\begin{array}{l}8.09 \\
8.84 \\
4.11 \\
5.27\end{array}$} & -13.37 & \multirow{2}{*}{$\begin{array}{l}42.03 \\
41.76\end{array}$} \\
\hline $08572+3915(a)$ & 5.7 & 61.3 & & -14.11 & \\
\hline $08572+3915$ (b) & 4.3 & 37.5 & & -14.43 & \multirow{2}{*}{$\begin{array}{l}40.73 \\
41.47\end{array}$} \\
\hline $\begin{array}{l}09143+0939 \\
09168+0623\end{array}$ & 7.6 & 41.0 & & -13.75 & \\
\hline $\begin{array}{l}09168+0623 \\
09192+2124\end{array}$ & 11.7 & $\begin{array}{r}313.9 \\
81.0\end{array}$ & \multirow{4}{*}{$\begin{array}{l}9.33 \\
9.44\end{array}$} & $\begin{array}{l}-13.21 \\
-13.85\end{array}$ & \multirow{3}{*}{$\begin{array}{l}42.34 \\
40.21\end{array}$} \\
\hline M82 & 28.6 & 153.9 & & $\begin{array}{l}-13.85 \\
-12.17\end{array}$ & \\
\hline NGC 3094 & & 12.3 & & -13.70 & \\
\hline $\begin{array}{l}10173+0828 \\
10485-1447\end{array}$ & & 19.9 & & & \\
\hline $10502-1843$ & & 47.4 & \multirow{5}{*}{$\begin{array}{r}11.75 \\
7.63 \\
7.76\end{array}$} & -13.30 & \multirow{5}{*}{$\begin{array}{l}42.51 \\
42.28 \\
43.63\end{array}$} \\
\hline $10565+2448$ & 7.9 & 75.0 & & -13.34 & \\
\hline $11095-0238$ & 12.0 & 96.9 & & -14.00 & \\
\hline $11119+3257$ & 49.5 & 197.0 & & -13.20 & \\
\hline $\begin{array}{l}11120-2919 \\
11122-2327\end{array}$ & & $\begin{array}{l}14.4 \\
72.1\end{array}$ & & -14.07 & \\
\hline NGC 3690 (a) & 62.7 & 300.4 & 3.42 & -12.39 & 41.06 \\
\hline NGC $3690(b)$ & 37.9 & 202.0 & \multirow[t]{3}{*}{6.27} & -12.72 & \multirow{4}{*}{41.29} \\
\hline NGC $3690(\mathrm{c})$ & & 172.0 & & & \\
\hline $11506-3851$ & & 26.5 & & & \\
\hline $12018+1941$ & & 28.3 & \multirow{3}{*}{$\begin{array}{r}10.05 \\
5.81\end{array}$} & -14.74 & \\
\hline $12112+0305$ & 7.8 & 65.2 & & -13.96 & \multirow{2}{*}{$\begin{array}{l}42.23 \\
40.77\end{array}$} \\
\hline $\begin{array}{l}\text { NGC } 4194 \\
\text { NGC } 4418\end{array}$ & 16.3 & $\begin{array}{r}126.0 \\
2.0\end{array}$ & & -12.37 & \\
\hline $13136+6223(\mathrm{a})$ & 43.1 & 257.9 & \multirow{3}{*}{$\begin{array}{l}5.91 \\
7.55 \\
9.90\end{array}$} & -12.80 & \\
\hline $13136+6223(\mathrm{~b})$ & 8.7 & 50.9 & & -13.79 & 41.44 \\
\hline $\begin{array}{c}\text { Mrk 273 } \\
14348-1447\end{array}$ & 11.1 & 90.0 & & -13.17 & 42.42 \\
\hline $15225+2350$ & 7.2 & 53.2 & 9.55 & -14.28 & 42.45 \\
\hline $15250+3609$ & & $\begin{array}{l}39.6 \\
28.6\end{array}$ & & & \\
\hline Arp 220 & & $\begin{array}{l}28.6 \\
94.1\end{array}$ & $14.40 \mathrm{~F}$ & & \\
\hline NGC 6240 & & 94.1 & $14.40 \mathrm{~F}$ & & \\
\hline $\begin{array}{l}17132+5313(a) \\
17132+5313(b)\end{array}$ & & $\begin{array}{r}93.1 \\
5.3\end{array}$ & & & \\
\hline $22491-1808$ & & & $7.94 \mathrm{E}$ & & \\
\hline $23488+2018$ & & 60.6 & & & \\
\hline *NGC 253 & & 50.5 & & & \\
\hline *NGC 660 & 2.9 & 22.8 & 14.07 & -13.67 & 40.03 \\
\hline *NGC 2146 & 9.4 & 66.7 & 13.66 & -12.98 & 40.70 \\
\hline *NGC 3079 & & & $6.33 G$ & $-13.72 \mathrm{G}$ & 39.50 \\
\hline $\begin{array}{l}{ }^{*} \text { NGC } 3256 \\
*_{\text {Mrk 266(a) }}\end{array}$ & & 143.5 & & & \\
\hline $\begin{array}{l}{ }^{*} \text { Mrk 266(a) } \\
\text { *Mrk 266(b) }^{2}\end{array}$ & 32.2 & 136.1 & 4.44 & -12.83 & 41.76 \\
\hline${ }^{*}$ Mrk 266(b) & 23.2 & 112.1 & 5.73 & -13.17 & 41.65 \\
\hline
\end{tabular}

Col. (2). - The observed equivalent width (in $\AA$ ) of the $\mathrm{H} \beta$ emission line. Col. (3). - The observed equivalent width (in $\AA$ ) of the $\mathrm{H} \alpha$ emission line.

Col. (4).-The observed ratio of the flux in the $\mathrm{H} \alpha$ emission line, to that in the $\mathrm{H} \beta$ emission line. line.

Col. (5).-The log of the observed flux (in ergs $\mathrm{cm}^{-2} \mathrm{~s}^{-1}$ ) of the $\mathrm{H} \alpha$ emission

Col. (6).-The $\log$ of the luminosity (in ergs $\mathrm{s}^{-1}$ ) of the $\mathrm{H} \alpha$ emission line, corrected for reddening using the observed $\mathrm{H} \alpha / \mathrm{H} \beta$ ratio in col. (4).

Notes.- * : These galaxies were selected by IR flux $\left(S_{60 \mu \mathrm{m}}>10 \mathrm{Jy}\right)$, and not IR color (see text). E: Data from Sanders et al. 1988; F: Data from Fosbury and Wall 1979; G: Data from Heckman, Balick, and Crane 1980 
found in the disk or nucler $\mathrm{H}$ II regions (see below), a correction for reddening would only serve to increase this disparity.

The equivalent width of the $\mathrm{H} \alpha$ emission line $\left(\mathrm{EQW}_{\mathrm{H} \alpha}\right)$ can also be used for comparison purposes between our FIRG nuclei and the $\mathrm{H}$ II regions of Kennicutt, Keel, and Blaha (1989). The $\mathrm{EQW}_{\mathrm{H} \alpha}$ essentially measures the ratio of ionizing photons to visible continuum photons from the imbedded source. For the nuclear $\mathrm{H}$ II regions, the median value of $\log$ $\mathrm{EQW}_{\mathrm{H} \alpha}$ is $\sim 1.5$, and for the disk $\mathrm{H}$ II regions the median value is $\sim 2.7$ (where $\mathrm{EQW}_{\mathrm{H} \alpha}$ is measured in $\AA$ ). Our FIRG nuclei have a median $\log \mathrm{EQW}_{\mathrm{H} \alpha}$ of $\sim 1.8$. These distributions can be seen in Figure 6. It is clear then, that while the $\mathrm{H} \alpha$ equivalent widths of our FIRG nuclei are slightly larger than those found in normal $\mathrm{H}$ in region nuclei, they are much smaller (by almost an order of magnitude) than those found in disk $\mathrm{H}$ II regions.

\section{b) Stellar Populations}

The optical spectra of old ( $>10^{9} \mathrm{yr}$ ) stellar populations are dominated by absorption lines due to neutral and lowionization metals and molecular absorption bands. One of the strongest and most easily measured of these features is the $\mathrm{Mg}$ I $b$ band at $\lambda \sim 5174 \AA$. The equivalent width $\left(\mathrm{EQW}_{\mathrm{Mg}}\right)$ of this band is listed in Table 2 for our galaxies. Using the KaplanMeier estimator, which takes explicit account of the upper limits (cf. Feigelson and Nelson 1985), we find that the mean value for the equivalent width is $\sim 0.8 \AA \pm 0.1 \AA$ for our FIRGs. A complete sample of optically bright galaxies from Heckman, Balick, and Crane (1980) and a complete sample of optically bright field spirals from Stauffer (1982) can be
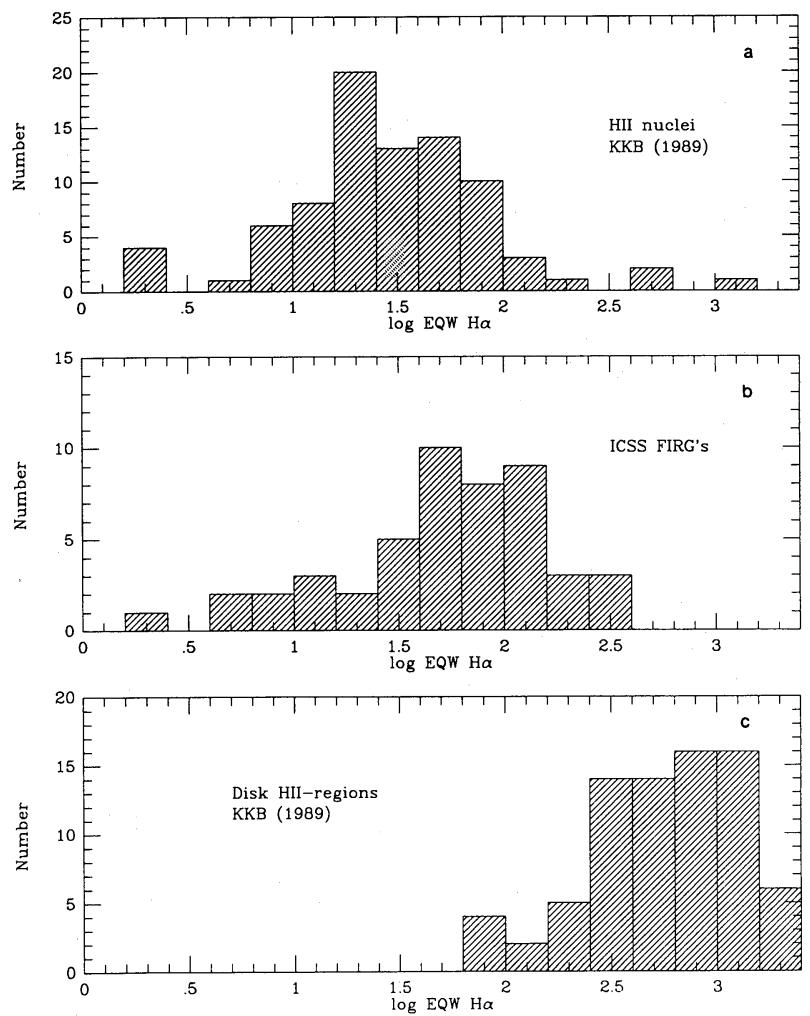

FIG. 6- (a) Distribution of the equivalent width of the $\mathrm{H} \alpha$ emission line for the H II region nuclei of Kennicutt, Keel, and Blaha (KKB 1989), (b) for all the ICSS FIRG nuclei, and (c) for the disk $\mathrm{H}$ II regions of Kennicutt, Keel, and Blaha (KKB 1989).
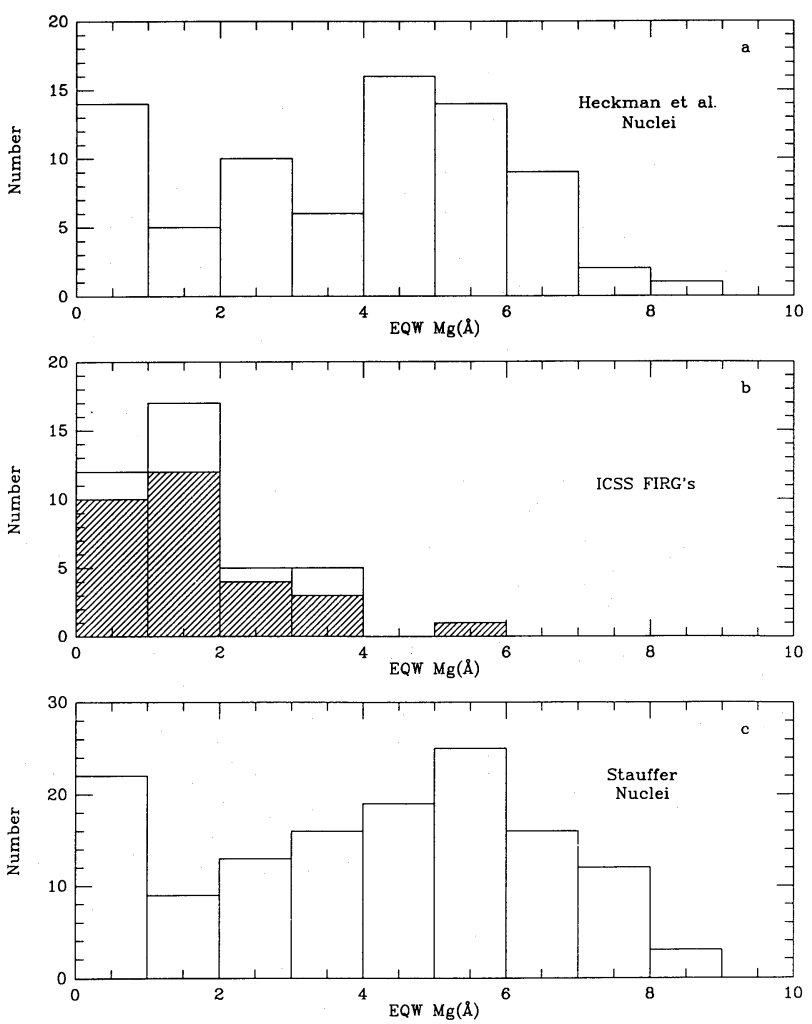

Fig. 7.- (a) Distribution of the equivalent width of the $\mathrm{Mg}$ I $\lambda 5174$ absorption line for the bright galaxy sample of Heckman, Balick, and Crane (1980), (b) for the ICSS FIRGs (shaded areas denote upper limits to the $\mathrm{Mg}$ I equivalent width), and (c) for the bright spiral galaxy sample of Stauffer (1982).

employed for comparison purposes. Figure 7 displays the distribution of $\mathrm{EQW}_{\mathrm{Mg}}$ for the Heckman, Balick, and Crane (1980) sample (mean value $~ 3.7 \AA$ ), the Stauffer (1982) sample (mean value $\sim 4.0 \AA$ ), and our infrared color-selected galaxies. Although there are fewer FIRGs, and many upper limits, it is clear that the nuclei of our program galaxies as a class have much lower values for the $\mathrm{Mg}$ equivalent width than do the nuclei of a sample of bright, otherwise normal galaxies.

The fact that the IR color-selected galaxies have unusually low values for $\mathrm{EQW}_{\mathbf{M g}}$ implies that the continuum is dominated by either a nonthermal power law, or a large number of hot, young stars. In the former case, the region of weak $\mathrm{Mg}$ I should be confined to the central most nucleus. To test this, we have measured the off-nuclear Mg equivalent width for 14 galaxies with good signal-to-noise ratio off the nucleus. In all cases the Mg I line remains weak at distances of $1-4 \mathrm{kpc}$ from the nucleus, with equivalent widths always below $2.5 \AA$, and in most cases, below 1.0 $\AA$. In fact, using the Kaplan-Meier estimator mentioned above, we find that the mean value of $\mathrm{EQW}_{\mathrm{Mg}}$ for the off-nuclear data of sufficient signal-to-noise is $\sim 0.7 \AA \pm 0.1 \AA$. This value is almost identical to that found for the nuclei themselves. The equivalent widths of the off-nuclear $\mathrm{Mg}$ then, do not approach the levels expected for a normal sample of galaxies. This kind of extended, weak $\mathrm{Mg}$ absorption could not be produced solely by a compact active nucleus, and it implies that vigorous star formation has occurred in most of the sample galaxies sometime during the past $\sim 10^{8} \mathrm{yr}$.

While the $\mathrm{Mg}$ I line is very weak in our nuclei, the $\mathrm{TiO}$ absorption band at $\sim 6250 \AA$ is typically quite strong (depth $\sim 5 \%-10 \%$ of the continuum). This combination of weak $\mathrm{Mg}$ I 
and strong $\mathrm{TiO}$ is reproduced in the models of Bica, Alloin, and Schmidt (1989) for poststarburst systems with ages $10^{7} \leq t \leq 10^{8} \mathrm{yr}$. In these models, the strong TiO absorption comes from red supergiants which dominate the red light at $t \geq 10^{7} \mathrm{yr}$.

Three of the galaxies listed in Table 2 exhibit strong Balmer absorption lines in their spectra. These galaxies are $01418+1651$, NGC 2623, and NGC 3094. As an example, the spectrum of NGC 2623 is presented in Figure 8. Because of the steep emission-line Balmer decrement, the lines of $\mathrm{H} \beta$ and $\mathrm{H} \gamma$ appear in absorption, while $\mathrm{H} \alpha$ appears in emission. These strong absorption features found in all three galaxies, indicate a large intermediate-age $\left(10^{8}-10^{9} \mathrm{yr}\right)$ population of stars. While Balmer absorption lines dominate the blue spectra of only these three galaxies, at least six other nuclei show evidence for strong Balmer absorption lines partially filled in by emission lines.

The presence of Wolf-Rayet emission features in the integrated spectrum of a galaxy is very rare, but it can provide important clues to the recent star formation history of the galaxy. In one of our program galaxies $(01003-2238)$, the lines of He I $\lambda 5876, \mathrm{C}$ III $\lambda 5696$, and $\mathrm{N}$ IV $\lambda 5737$, as well as a broad feature centered at $4660 \AA$ made up of $\mathrm{He}$ II $\lambda 4686$, and N III $\lambda \lambda 4634,4640,4642$ have been detected. Here, these emission lines are believed to be due to the combined effects of approximately $3 \times 10^{5}$ Wolf-Rayet stars of the WN subclass (Armus, Heckman, and Miley 1988). The ratio of the number of WolfRayet stars to massive OB stars in $01003-2238$ is $\sim 0.53$. This value, over 10 times that found in the Milky Way, is used to argue for a steeply declining star formation rate in $01003-2238$ over the past $10^{7}$ yr (Armus, Heckman, and Miley 1988).

Four other sample nuclei (NGC 1222, NGC 1614, NGC 3690 nucleus a, and NGC 4194) have weak, broad emission centered at $4660 \AA$ (rest frame). This broad emission is composed of at least three lines. The redmost line (FWHM $\sim 1000$ $\mathrm{km} \mathrm{s}^{-1}$ ) can be positively identified as He II $\lambda 4686$. The bluemost feature, at a rest wavelength of $\lambda_{0} \sim 4628 \AA$, has a similarly large line width and may have a contribution from $N$ III or $\mathrm{N} \mathrm{v}$, or be a blend of at least two nebular lines. The middle of the three lines has a FWHM of $\leq 400 \mathrm{~km} \mathrm{~s}^{-1}$, and a rest wavelength of $\lambda_{0} \sim 4657 \AA$. This line cannot be identified with

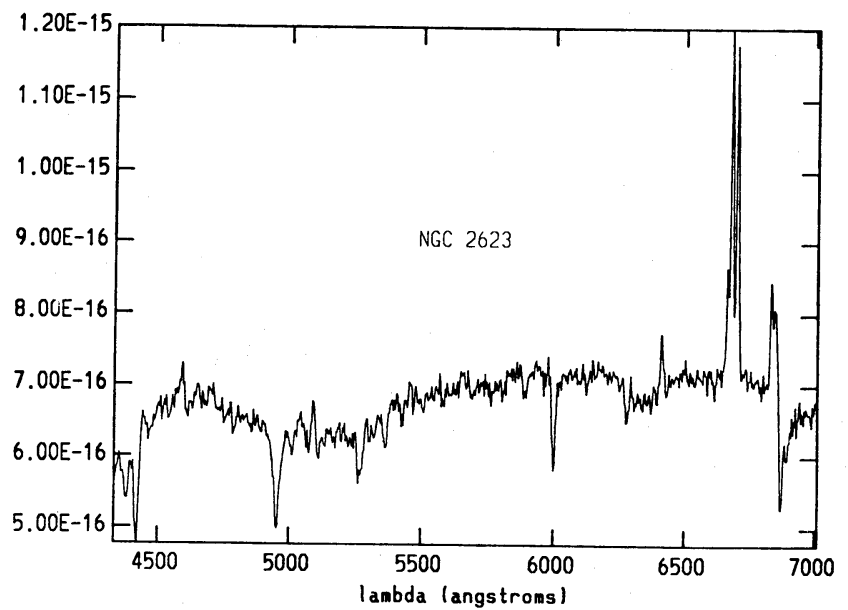

FIG. 8.-Flux-calibrated nuclear spectrum of NGC 2623 (vertical scale is in ergs $\left.\mathrm{cm}^{-2} \mathrm{~s}^{-1} \AA^{-1}\right)$. The strongest feature is the $\mathrm{H} \alpha,[\mathrm{N}$ II] blend. Note the strong Balmer absorption lines.
TABLE 5

WOLF-RAYET FEATURES

\begin{tabular}{|c|c|c|c|c|}
\hline $\begin{array}{l}\text { Galaxy } \\
\text { (1) }\end{array}$ & $\begin{array}{c}\mathrm{EQW}_{\lambda 4660} \\
\text { (2) }\end{array}$ & $\begin{array}{c}F_{\lambda 4660} \\
\text { (3) }\end{array}$ & $\begin{array}{c}L_{\lambda 4660} \\
(4)\end{array}$ & $\begin{array}{l}L_{\mathrm{WR}} / L_{\mathrm{H} \beta} \\
(5)\end{array}$ \\
\hline $01003-2238$ & 15.0 & -15.07 & 41.46 & 0.64 \\
\hline NGC $1222 \ldots \ldots \ldots \ldots$ & 2.1 & -14.11 & 40.32 & 0.13 \\
\hline NGC 1614 & 2.1 & -14.40 & 40.88 & 0.08 \\
\hline NGC 3690(a) & 3.7 & -14.11 & 39.43 & 0.07 \\
\hline NGC $4194 \ldots$ & 1.2 & -14.24 & 39.26 & 0.09 \\
\hline
\end{tabular}

Col. (2). - The observed equivalent width (in $\AA$ ) of the broad Wolf-Rayet emission feature centered at a rest wavelength of $4660 \AA$

Col. (3). - The log of the observed flux (in ergs $\mathrm{cm}^{-2} \mathrm{~s}^{-1}$ ) of the $4660 \AA$ emission feature.

Col. (4). - The log of the luminosity (in ergs $\mathrm{s}^{-1}$ ) of the $4660 \AA$ emission feature, corrected for reddening using the observed $\mathbf{H} \alpha / \mathrm{H} \beta$ ratio

Col. (5).- The ratio of the luminosity of the $4660 \AA$ emission feature to that of the $\mathrm{H} \beta$ emission line.

any known Wolf-Rayet emission feature, and is probably of nebular origin (possibly Ar II or Fe II). The equivalent width, the flux, and the luminosity of the total $4660 \AA$ emission feature, as well as its ratio to $L_{\mathbf{H} \beta}$, is listed in Table 5 for all five nuclei. An identification of the $\lambda_{0} \sim 4628 \AA$ feature, as well as higher signal-to-noise ratio data in other parts of the spectrum containing Wolf-Rayet emission lines, will be required before a statement can be made concerning the star formation histories of NGC 1222, NGC 1614, NGC 3690, and NGC 4194

\section{c) Reddening}

Reddening corrections were derived from the observed $\mathbf{H} \alpha / \mathbf{H} \beta$ flux ratio for all objects with flux calibrated spectra. An intrinsic ratio of $\mathrm{H} \alpha / \mathrm{H} \beta=2.86$ was assumed, and a standard interstellar extinction curve (Osterbrock 1988) was used. Flux corrections derived from these values will generally be underestimates, because in the likely situation of the dust and gas being mixed, the true extinction will be higher than that implied by the Balmer decrement. We can compare this firstorder estimate of the reddening in our sample galaxies, to that found for other groups of active galaxies by Dahari and De Robertis (1988), and for nuclear and disk H II regions by Kennicutt, Keel, and Blaha (1989). The parameter $c_{1}=$ $2.86\left[\log \left(R_{\mathrm{obs}} / R_{\mathrm{int}}\right)\right]$ is calculated, where $R_{\mathrm{obs}}$ is the observed Balmer decrement, corrected for underlying stellar absorption, and $R_{\mathrm{int}}$ is the intrinsic $\mathrm{H} \alpha / \mathrm{H} \beta$ ratio, taken either as 2.86 for ionization by starlight, or as 3.1 for power-law photoionization. Note that $c_{1}$ is the extinction in $\log _{10}$ units at $\mathrm{H} \beta$. For Seyfert 1 galaxies $c_{1}=0.63$, for Seyfert 2 galaxies $c_{1}=0.93$, for LINERs $c_{1}=1.05$, and for Markarian starburst galaxies $c_{1}=$ 1.01. In arriving at these averages, we have taken Seyfert 1-1.5 galaxies as Seyfert 1's, and Seyfert 1.8-2.0 galaxies as Seyfert 2's. For comparison our sample of infrared color-selected FIRGs has an uncorrected mean value of $c_{1}=1.20$.

An underlying Balmer absorption of stellar origin will steepen the measured Balmer decrement, causing the extinction corrections to be overestimated. Since this absorption is the same at $\mathrm{H} \alpha, \mathrm{H} \beta$, and $\mathrm{H} \gamma$ to within $30 \%$ (Kurucz 1979), we have used a common absorption equivalent width to correct the measured $\mathrm{H} \alpha$ and $\mathrm{H} \beta$ emission lines. The absorption at $\mathrm{H} \beta$ has been measured as $\sim 2 \AA$ in a sample of extragalactic H II regions by McCall, Rybski, and Shields (1985). Similar values of 1-2 $\AA$ are seen in older stellar populations whose integrated optical light is dominated by G-K giants (Kennicutt 1983). The A stars, which have the largest Balmer equivalent widths 
along the spectral sequence, typically have values of $\sim 4 \AA$ at $\mathrm{H} \beta$ as giants, and $\sim 8 \AA$ at $\mathrm{H} \beta$ as dwarfs (O'Connell 1973). Taking a value of $3 \AA$ at $\mathrm{H} \alpha$ and $\mathrm{H} \beta$ (intermediate between a young or old population, and giant A stars), and correcting the observed Balmer decrement for this absorption, we arrive at a new average extinction for our ICSS FIRGs of $c_{1}=0.97$. The median values of $c_{1}$ for the samples of Kennicutt, Keel, and Blaha (1989), are $\sim 0.67$ for the $\mathrm{H}$ il region nuclei, and $\sim 0.47$ for the disk $\mathrm{H}$ II regions. The emission-line gas in our ICSS FIRGs then, is reddened at $\mathbf{H} \beta$ by an amount $\sim 3$ times that observed for normal disk $H$ II regions, and $\sim 2$ times that seen in nuclear $\mathrm{H}$ II regions. This is not surprising, given the large amounts of dust implied by high values of $L_{\mathrm{FIR}}$, and $L_{\mathrm{FIR}} / L_{\mathrm{B}}$ measured for our ICSS FIRGs.

Many of the galaxies in our sample have a strong $\mathrm{Na}$ I $\mathrm{D}$ absorption feature at $\lambda \sim 5893 \AA$. While this line may in general have either an interstellar or a stellar atmospheric origin, the former is likely to be dominant in our galaxies, as we now show. While the nuclei of early-type galaxies generally have strong $\mathrm{Na} \mathrm{D}$ absorption, this feature arises predominantly in the photospheres of stars, with a strength that is well correlated with other photospheric lines like the $\mathrm{Mg}$ I $b$ band. The situation in the FIRGs is quite different. First, the $\mathrm{Na} \mathrm{D}$ and $\mathrm{Mg} b$ equivalent widths are not correlated (see Fig. 9). Second, the strength of the $\mathrm{Na} \mathrm{D}$ line in the FIRGs is well

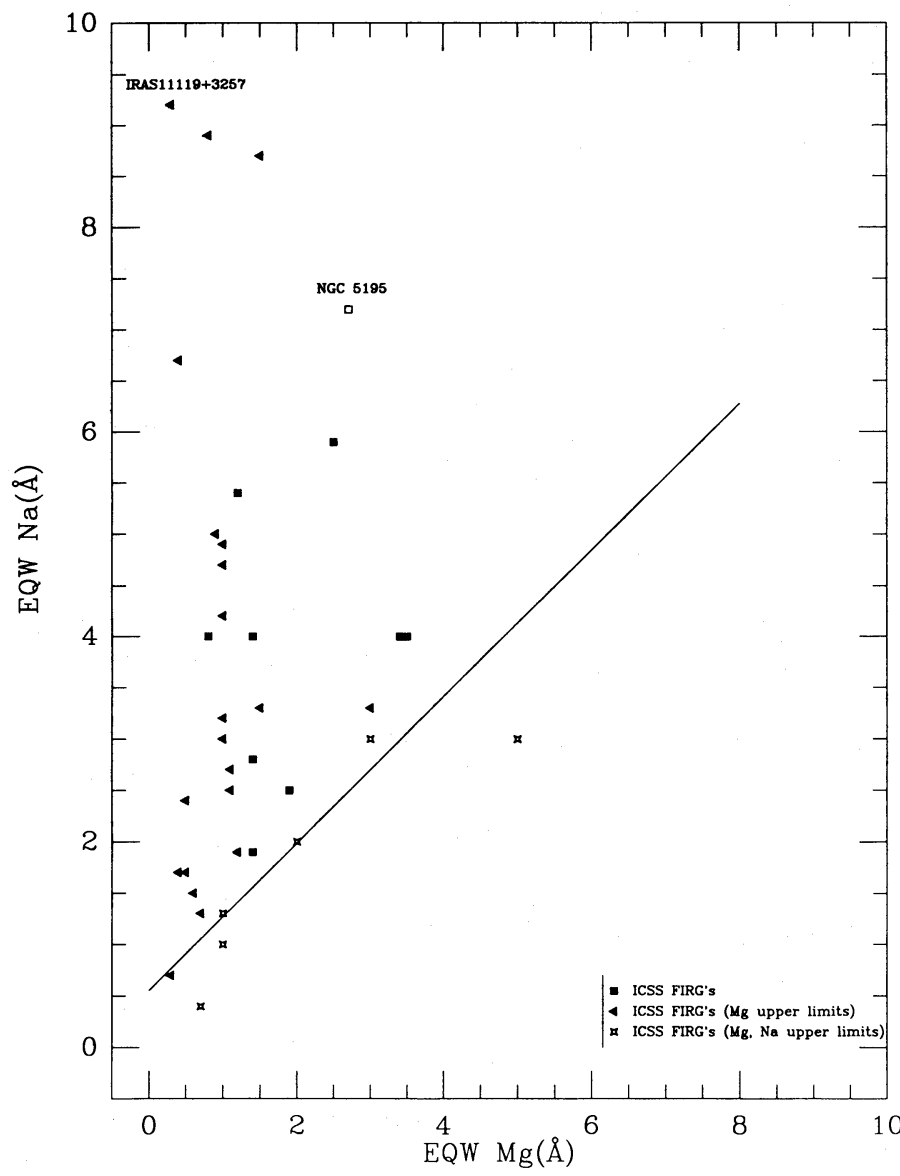

FIG. 9.-Plot of the equivalent width of the $\mathrm{Na}$ I $\mathrm{D}$ absorption lines against the equivalent width of the $\mathrm{Mg}$ I absorption line for the ICSS FIRGs. The solid line is the linear best fit to the bright galaxy sample of Heckman, Balick, and Crane (1980), excluding the galaxy NGC 5195 (the unusually dusty companion to M51). This galaxy is denoted by an open square and labeled.

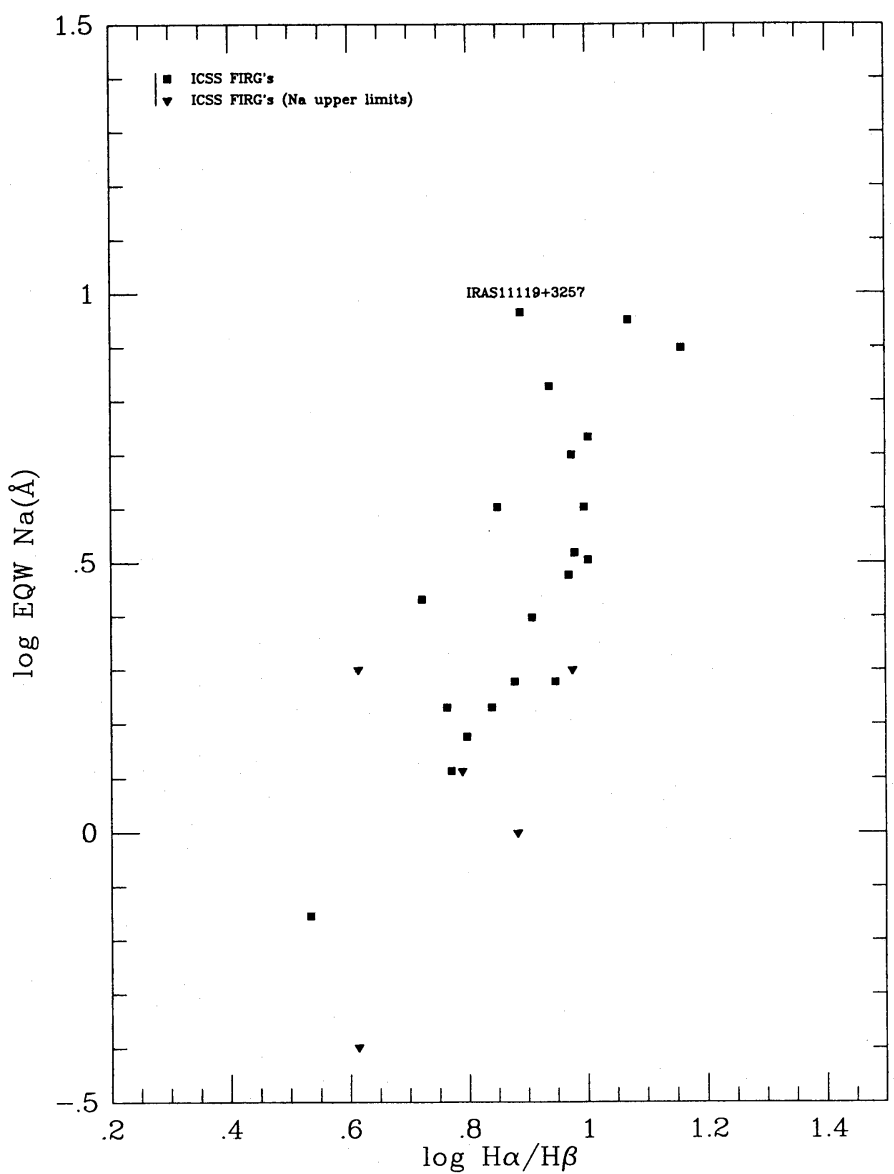

Fig. 10.-Plot of the equivalent width of the $\mathrm{Na}$ I $\mathrm{D}$ absorption lines against the observed Balmer decrement for the ICSS FIRGs.

correlated with the amount of extinction indicated by the Balmer decrement (see Fig. 10). We therefore conclude that the strong $\mathrm{Na} \mathrm{D}$ lines in the FIRGs arise from an abnormally large column density of $\mathrm{Na} \mathrm{I}$ along the line of sight in the interstellar medium of the FIRGs. In theory, the ratio of the Na D $\lambda 5890$ and $\lambda 5896$ lines can be used to derive the column density and velocity dispersion of the neutral sodium atoms (Strömgren 1948). Unfortunately, the doublet is not resolved in our fluxcalibrated spectra and is just barely split in our high-resolution data.

As a final reddening indicator, we have measured the ratio of the continuum flux densities $\left(F_{\lambda}\right)$ at rest wavelengths equal to $6500 \AA$ and $4800 \AA$, both determined visually over $\sim 100 \AA$ wide bins (see Table 2). The values of the ratio for the IR color-selected sample range from $0.60-1.83$, with an average value of $C 65 / C 48=1.14$. Almost $75 \%$ of the galaxies have $C 65 / C 48>1.0$. In normal galactic nuclei, the color of the continuum primarily measures the age of the stellar population. For such nuclei, red colors are well correlated with strong stellar absorption lines from an old population (e.g., the $\mathbf{M g}$ I $b$ band). In contrast, the FIRGs have red continua, plus weak $\mathrm{Mg}$ I (see Fig. 11). Specifically, a galaxy with an $\mathrm{Mg}$ equivalent width of $0.8 \AA$, the average value for the sample, would have a $C 65 / C 48=0.78$ if placed on the linear best-fit line through the normal galaxy data of Heckman, Balick, and Crane (1980) in Figure 11. The highly reddened galaxy NGC 5195 (an extremely dusty companion to M51) has been left out of the fit 


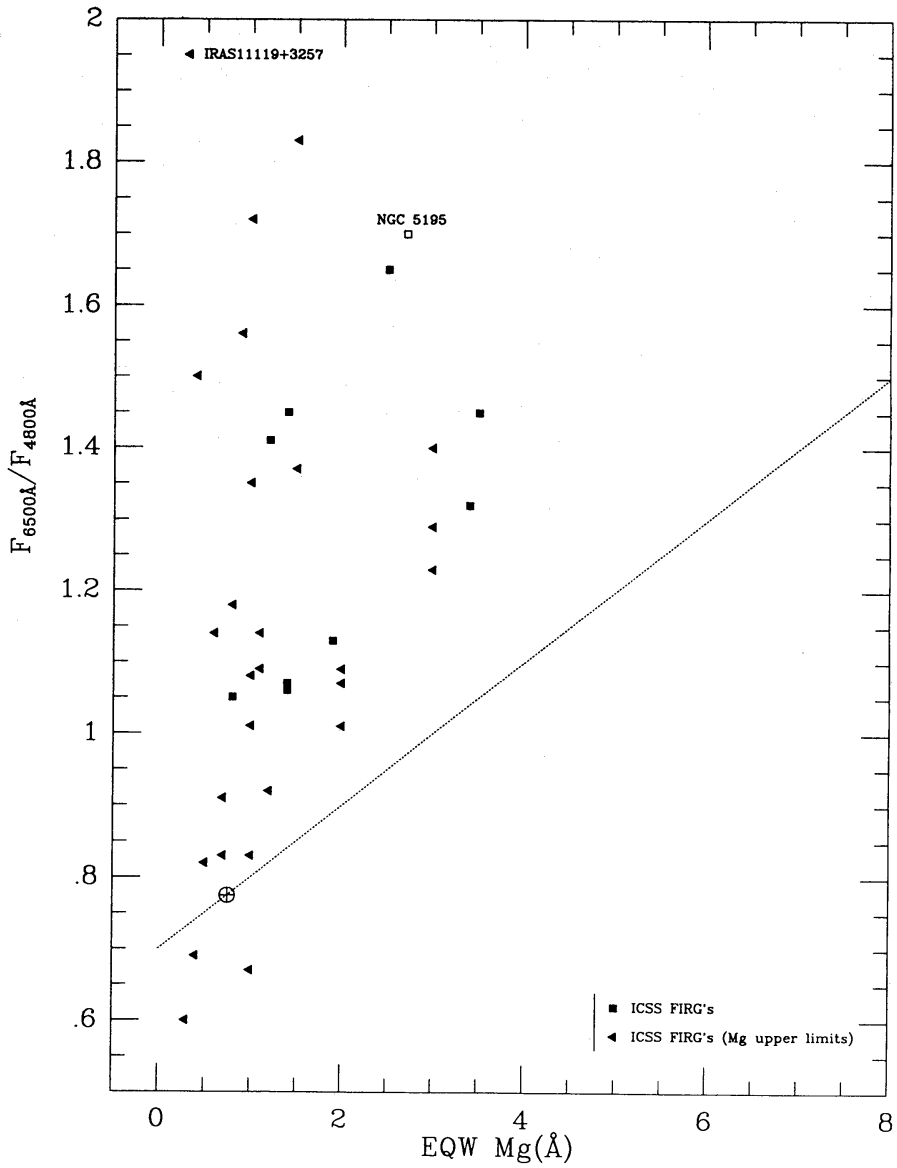

FIG. 11.-Plot of the ratio of the flux densities (ergs $\mathrm{cm}^{-2} \mathrm{~s}^{-1} \AA^{-1}$ ) in the continuum at $6500 \AA$ and $4800 \AA$ vs. the equivalent width of the $\mathrm{Mg}$ I $b$ band at $5174 \AA$ for the ICSS FIRGs. The solid line is the linear best fit to the bright galaxy sample of Heckman, Balick, and Crane (1980), excluding the galaxy NGC 5195 (the unusually dusty companion to M51). This galaxy is denoted by an open square and labeled. The crosshairs represent the position an ICSS FIRG would occupy on this line, if it had a value for $\mathrm{EQW}_{\mathrm{Mg}}$ equal to the average for the entire sample $(0.8 \AA)$.

and is labeled in Figure 11. Our infrared color-selected galaxies are then unusually red for their weak $\mathrm{Mg}$ equivalent widths, as expected if they suffer heavy interstellar extinction. In further support of this hypothesis, we find a good correlation between red colors and both steep Balmer decrements and strong $\mathrm{NaD}$ absorption for the FIRGs (see Fig. 12).

We also note that Mirabel and Sanders (1988) find that the $\mathrm{H} \mathrm{I} 21 \mathrm{~cm}$ line is seen in absorption against the nuclear radio source in about half of all FIRGs with $L_{\text {FIR }} \geq 10^{11} L_{\odot}$. The implied $\mathrm{H}$ I column densities are typically $1-5 \times 10^{21} \mathrm{~cm}^{-2}$ for a spin temperature of $100 \mathrm{~K}$. Assuming a standard value for the gas-to-dust ratio, this would correspond to typically $A_{V} \sim$ 0.6-3 mag. The corresponding values for $c_{1}$ (see above) would be $0.3-1.4$. Such values are consistent with those we derive from the Balmer decrement in our infrared color-selected FIRGs. Unfortunately we have too few FIRGs in common with Mirabel and Sanders to look for any correlation between $\mathrm{H}$ I column density and our colors, Na D strengths, or Balmer decrements.

\section{DISCUSSION}

a) The Nature of the Power Source

The range in nuclear properties exhibited by our ICSS FIRGs raises two important questions. The first of these con- cerns the nature of the power source behind the optical and infrared radiation and can be stated as follows.

Is the dust and emission-line gas in these galaxies powered entirely by a starburst, or is a nonthermal ionization source required? The relative emission-line strengths, plotted in Figures $1 a$ and $1 b$, imply that only about one-third to one-half of the FIRGs (namely those with spectra similar to giant $\mathrm{H}$ II regions) can be understood in terms of photoionization by normal OB stars. The others require an alternative, or additional source of ionizing photons (with the two most plausible possibilities being either an AGN, or shocks associated with a starburst-driven "superwind"). To assess the possibility that a starburst alone can account for the properties of nearly all of the ICSS FIRGs, we first summarize the evidence for a dominant young stellar population in all of our FIRGs. We will then try to estimate the luminosity associated with this young stellar population, assuming that a lower limit to the extinction correction appropriate to the optical continuum is provided by the Balmer decrement (a procedure for which we will provide some empirical support).

The best evidence that the optical continuum is dominated by young stars comes from the fact that the spectroscopic signatures of an old population (e.g., the $\mathrm{Mg} \mathrm{I} b$ stellar absorption line) are weak not only in the nuclei, but out to distances of several $\mathrm{kpc}$ from the nucleus (an AGN alone would greatly weaken the $\mathrm{Mg} b$ line only in the nucleus). The presence of strong Balmer absorption lines, and broad emission features

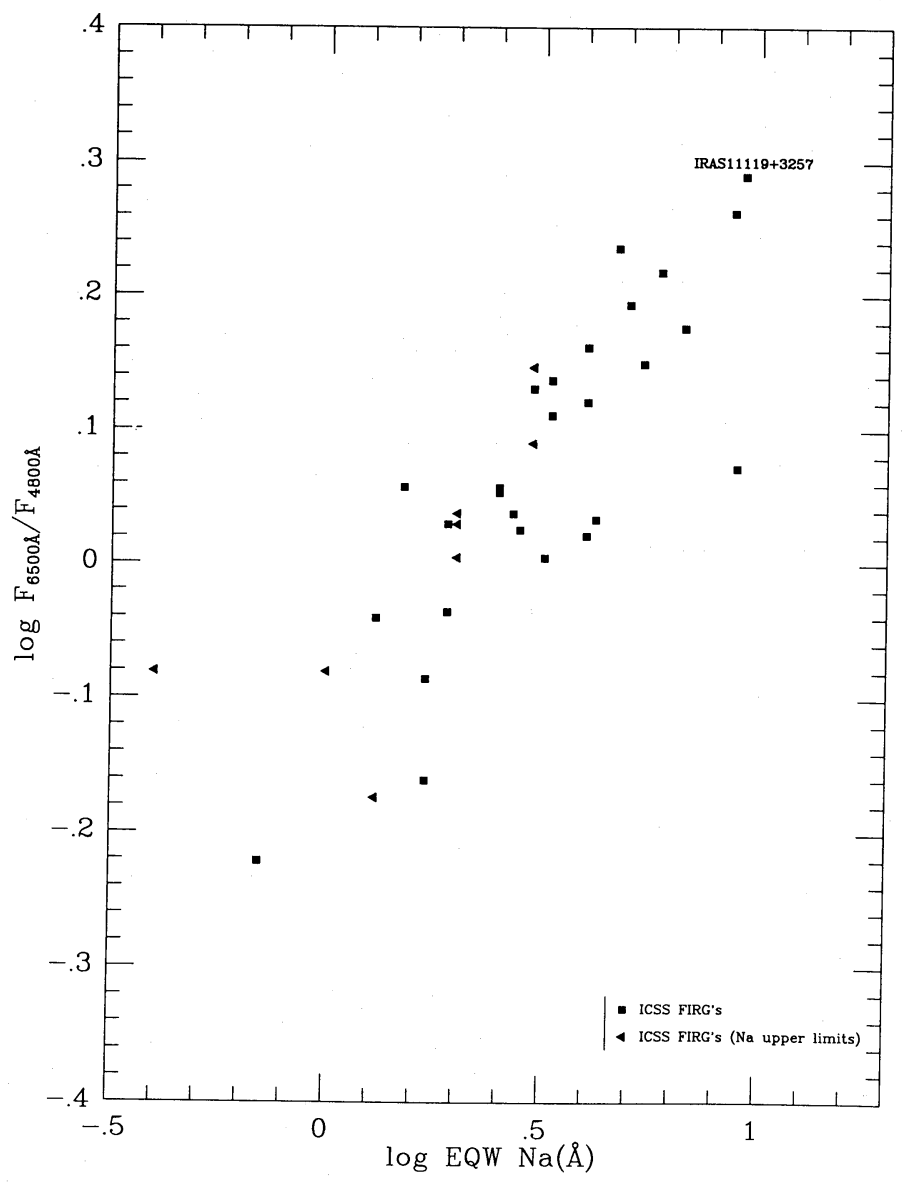

Fig. 12.-Plot of the ratio of the flux densities (ergs cm $\left.\mathrm{cm}^{-2} \mathrm{~s}^{-1} \AA^{-1}\right)$ in the continuum at $6500 \AA$ and $4800 \AA$ vs. the equivalent width of the $\mathrm{Na}$ I D absorption lines for the ICSS FIRGs. 
attributed to Wolf-Rayet stars, is additional strong evidence for large populations of intermediate-age and young stars, respectively, in at least some of the ICSS FIRGs. For one galaxy in particular, namely $01003-2238$, a very high ratio of Wolf-Rayet to $\mathrm{O}$ stars implies a steeply declining rate of star formation within the last $\sim 10^{7} \mathrm{yr}$ (Armus, Heckman, and Miley 1988). Four other FIRGs in our sample also probably have a large Wolf-Rayet population.

As mentioned in $\S$ III, the equivalent width of the $\mathrm{H} \alpha$ emission line, which measures the ratio of ionizing to optical continuum radiation, can be used to set a limit on the age of the stellar population responsible for heating the gas. The median value of $\log \mathrm{EQW}_{\mathrm{H} \alpha}$ (where $\mathrm{EQW}_{\mathrm{H} \alpha}$ is measured in $\AA$ ) for our IR color-selected FIRGs is $\sim 1.8$. This is almost a factor of 10 down from that seen in a sample of disk $\mathrm{H}$ II regions by Kennicutt, Keel, and Blaha (1989). We can use the model calculations of $\mathrm{EQW}_{\mathbf{H} \boldsymbol{\beta}}$ from DeGioia-Eastwood (1985), coupled with the continuum colors of stellar cluster models from Jacoby, Hunter, and Christian (1984), to calculate $\mathrm{EQW}_{\mathbf{H} \alpha}$ for a ZAMS, and $10^{7} \mathrm{yr}$ old cluster formed with a Miller-Scalo (1979) IMF. This can then be used as a comparison to our value for the ICSS FIRG nuclei. This conversion from EQW to $\mathrm{EQW}_{\mathrm{H} \alpha}$ is independent of reddening as long as the emissionline gas and the continuum are reddened by the same material. For the ZAMS population, $\log \mathrm{EQW}_{\mathrm{H} \alpha} \sim 2.7$, and for the $10^{7}$ $\mathrm{yr}$ old population, $\log \mathrm{EQW}_{\mathrm{H} \alpha} \sim 1$.6. The $\mathrm{H} \alpha$ emission and optical continuum of our ICSS FIRGs then can be modeled as being produced either by a single population with an age slightly less than $\sim 10^{7} \mathrm{yr}$, or through the combination of an even younger population (such as those responsible for galactic disk $H$ II regions) and a population of intermediate age $\left(10^{8}-\right.$ $10^{9} \mathrm{yr}$ old) stars. These stars would presumably have been created earlier in the history of the starburst.

We can compare the average $C 65$-to- $C 48$ ratio and equivalent width of the Mg I absorption line in our ICSS FIRGs to the integrated spectra models of Bica, Alloin, and Schmidt (1989). When this is done, we find that both $C 65 / C 48$ (dereddened using the absorption-corrected Balmer decrement; see below) and $\mathrm{EQW}_{\mathrm{Mg}}$ best match the models having a burst-to-galaxy mass ratio of $\geq 10 \%$ and a time since the burst of $\leq \mathrm{a}$ few $\times 10^{7} \mathrm{yr}$. Our low EQW $\mathrm{Mg}_{\mathrm{Mg}}$ can also be reproduced by a young $\left(\sim 10^{7} \mathrm{yr}\right)$ population in which the weak $\mathrm{Mg} \mathrm{I}$ is produced by red supergiants, without the need for an underlying old preexisting population.

Figure 13 shows the $\log$ of the $C 65$ to $C 48$ ratio plotted against the Balmer decrement for our ICSS galaxies. We have also plotted the locus of the colors expected for four stellar populations (with ages of zero, $10^{7}, 10^{9}$, and $10^{10} \mathrm{yr}$ ) reddened by amounts given by the Balmer decrement and assuming case $B$ conditions. The intrinsic colors of these populations were taken from Jacoby, Hunter, and Christian (1984). While an assumption that the continuum and the line-emitting gas are reddened by the same amount may not be strictly true for our galaxies, the correlations of $\mathrm{H} \alpha / \mathrm{H} \beta$ with $C 65 / C 48$, and with the equivalent width of the $\mathrm{Na} \mathrm{D}$ doublet (see Figs. 10,12, and 13) is evidence the source of reddening is common to both the stars and the emission-line gas. Although the number of points in Figure 13 is low, all the galaxies fall well below (to the younger side of the reddening line of a $10^{10} \mathrm{yr}$-old stellar population and generally fall between the loci of a reddened ZAMS, and a $10^{9}$ yr-old stellar population. These results are consistent with the idea that many of our far-infrared color-selected galaxies contain predominantly a young stellar population, viewed through heavy extinction.

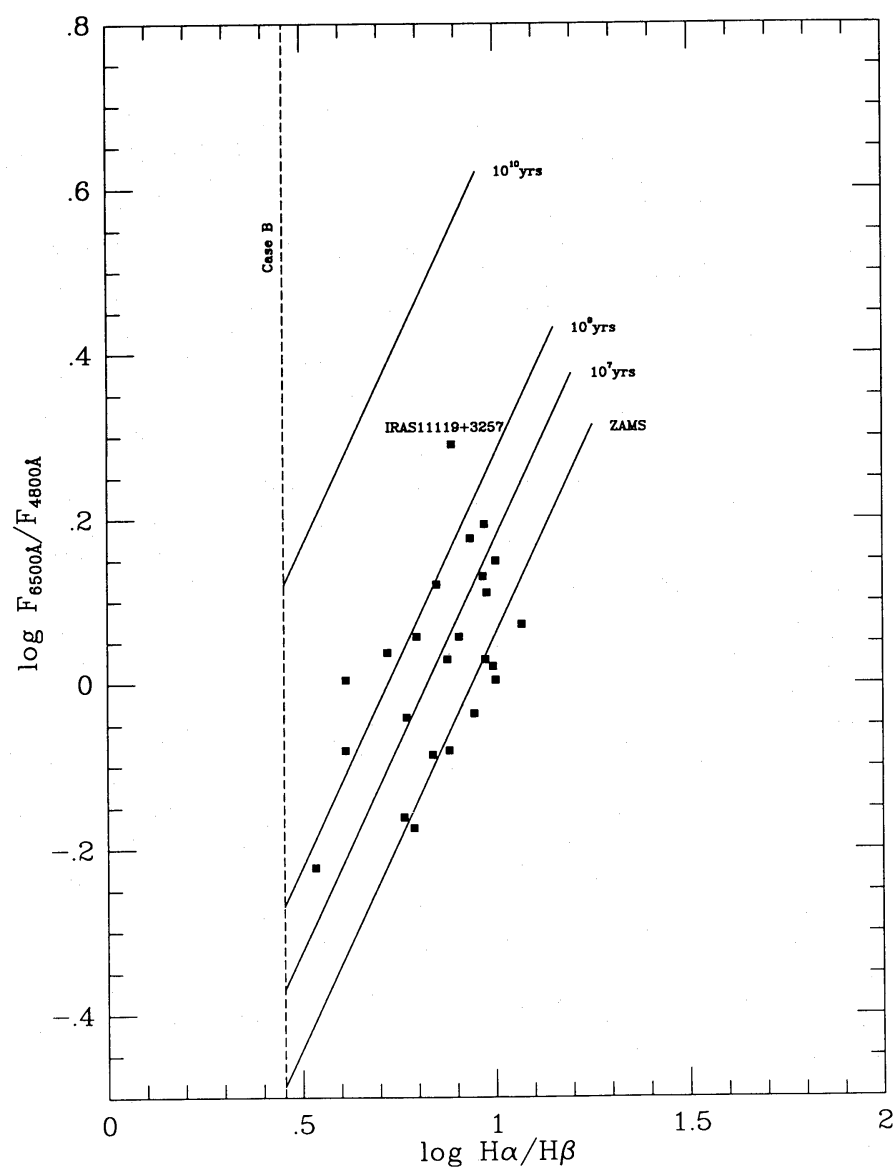

Fig. 13.-Plot of the ratio of the flux densities (ergs $\mathrm{cm}^{-1} \mathrm{~s}^{-1} \AA^{-1}$ ) in the continuum at $6500 \AA$ and $4800 \AA$ vs. the observed Balmer decrement for the ICSS FIRGs. The solid lines are the reddening vectors for star clusters of various ages as constructed by Jacoby, Hunter, and Christian (1984).

A quantitative evaluation of the energetic importance of the heavily reddened young stellar population is hampered by the uncertain extinction correction applicable to these stars. Simply correcting the observed luminosity using the Balmer decrement and assuming a normal total to selective extinction, corresponds to the physically implausible situation in which the emission-line gas and young stars are viewed through a uniform foreground dust screen. In the more realistic case in which the ionized gas, young stars, and dust are mixed (and distributed in a highly clumpy fashion), the extinction measured with the Balmer decrement will only be a lower limit to the true extinction since the observed emission lines and starlight arise preferentially in the least heavily reddened material. Nevertheless, it is still instructive to use the Balmer decrement to measure a lower limit to the true extinction, and thus a lower limit to the luminosity of the visible stellar population in our FIRGs.

For all the FIRGs with flux-calibrated spectra, we have therefore used the observed Balmer decrement and optical flux density to determine a lower limit to the extinction-corrected optical luminosity of the nucleus, parameterized as $L_{48}=\lambda P_{\lambda}$ (where $P_{\lambda}$ is the monochromatic power at $4800 \AA$ corrected for extinction using the Balmer decrement). We then find that the median value of $\log \left(L_{48} / L_{\text {FIR }}\right) \sim-1.0$ with typical values lying between -0.5 and -2.0 . If we use the Balmer decrement corrected for an underlying absorption of $3 \AA$ equivalent width (see above), the median value $\log \left(L_{48} / L_{\text {FIR }}\right) \sim-1.1$. The bolo- 
metric (total IR) luminosity will be somewhat larger than $L_{\mathrm{FIR}}$, which measures only the luminosity between $40 \mu \mathrm{m}$ and 120 $\mu \mathrm{m}$ (e.g., Rice et al. 1988 give a ratio of $L_{\mathrm{BOL}} / L_{\mathrm{FIR}}=2$ for M82 which has the prototypical spectral energy distribution for our ICSS FIRGs). Thus, the median lower limit to the ratio of the optical-to-bolometric luminosity in the nuclei of our FIRGs is $\sim 5 \%$ with the large scatter in our computed ratios being almost certainly a reflection of our overly simplistic method of correcting for extinction. For comparison, the models of Wyse (1985) yield values of $L_{48}$ that are about $20 \%$ of the bolometric luminosity for a stellar population with an age appropriate to a starburst $\left(10^{6}-10^{7} \mathrm{yr}\right)$. We conclude, then, that the young stellar population visible in the optical can typically provide at least $25 \%$ of the energy needed to power the far-infrared emission. In other words, we are seeing about one-fourth of the optical luminosity that one would expect from a population of young stars, given the observed far-infrared emission. Since we have probably substantially underestimated the extinction, the young stars visible optically may be adequate to power all the far-infrared emission. ${ }^{2}$

\section{b) The Nature of the Emission-Line Gas in FIRGs}

The second major question that presents itself is whether or not the color-selected FIRGs are indeed one class of object. If all of the program galaxies are of a single class, then why do major diagnostics such as the nuclear emission-line flux ratios and line widths not reflect this unity? A clue to the resolution of this ambiguity may be found through an investigation of the nearest member of the sample, M82.

In M82, the galaxy for which we have the highest spatial resolution, [ $\left.\mathrm{O}_{\mathrm{I}}\right] / \mathrm{H} \alpha$ changes from values like those seen in $\mathrm{H}$ II regions in the nucleus, to those more representative of shockheated gas at radii $\sim 1 \mathrm{kpc}$ from the nucleus (see Fig. 2). Also, the $\mathrm{H} \alpha \mathrm{FWHM}$ changes from $\sim 100 \mathrm{~km} \mathrm{~s}^{-1}$ in the nucleus, to typically $250-300 \mathrm{~km} \mathrm{~s}^{-1}$ at $r>400 \mathrm{pc}$. This type of ionization structure can be understood in the context of the starburstinduced, supernovae-driven wind model that has been proposed for this galaxy (Chevalier and Clegg 1985; McCarthy, Heckman, and van Breugel 1987; Bland and Tully 1988). In this model, the nuclear starburst would dominate the spectrum at small radii, and shock-heated emission-line gas would dominate the spectrum at large radii. If the other IR color-selected galaxies are of the same basic type as M82, then we might expect similar processes to be occurring, and similar spatial variations in the emission-line flux ratios and line widths to be observed. The spread seen in Figures $1 a, 1 b$, and 4 would then be (at least in part) the effect of using an aperture with a strongly redshift-dependent size for the measurements of the "nuclear" spectra. Since we would be sampling more of the off-nuclear gas for the more distant galaxies, this gas (if it is shock heated as in M82) would cause our "nuclear" emissionline flux ratios to move out of the domain of the $\mathrm{H}$ II regions in the diagnostic diagrams.

To check this possibility, we have compared the projected beam radius (the distance, in pc, along the slit which was collapsed to form the one-dimensional spectra) for our sample galaxies that fall in the AGN or LINER domain of Figure 1, to those that fall in the $\mathbf{H}$ II region domain. These two distributions can be seen in Figures $14 a$ and $14 b$. It is clear from this

${ }^{2}$ We note that in the two well-studied FIRGs M82 and NGC 3690, the lower limit to $L_{48} / L_{\mathrm{BOL}}$ is only $0.5 \%$ (compared to our median value of $5 \%$ ). In these galaxies at least, the actual energy source is evidently well shrouded.
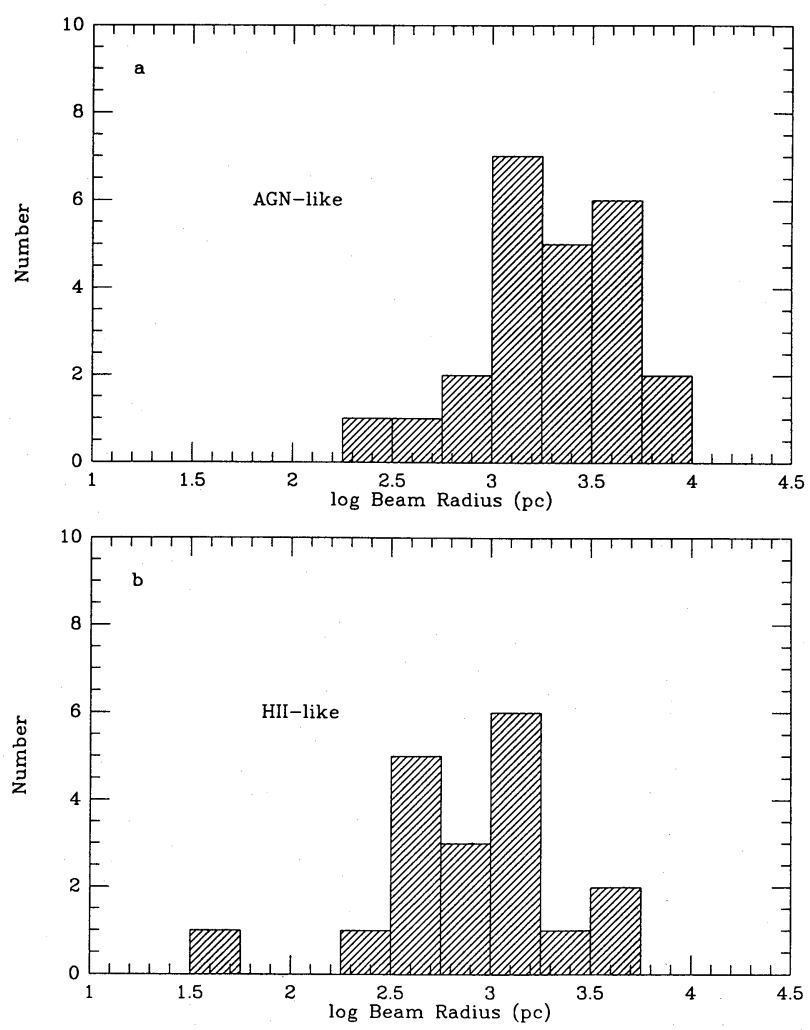

FrG. 14.-(a) Distribution of the projected beam size for the FCSS FIRGs with nuclear spectra resembling AGN (LINERs) and (b) for the FCSS FIRGs with nuclear spectra resembling $\mathbf{H}$ in regions.

figure that the AGN-like galaxies have been observed with significantly larger typical beam sizes (essentially because they are on average, about twice as distant as the $\mathrm{H}$ H region-like sample galaxies). While this result supports the above hypothesis of aperture-dependant line ratios, it does not prove the case. For an IR-flux-limited sample like ours, the distances and luminosities of the galaxies are correlated. Indeed, the AGNlike galaxies have greater redshifts (average $z \sim 0.08$ vs. $\sim 0.03$ ) and luminosities (log average $L_{\text {FIR }}\left(L_{\odot}\right)=11.8$ vs. log average $L_{\text {FIR }}\left(L_{\odot}\right)=11.3$ ) than the $\mathbf{H}$ II region-like galaxies. ${ }^{3}$ In fact, six of the seven IR color-selected galaxies with $\log L_{\text {FIR }} \geq 12.0 L_{\odot}$ are AGN-like. An alternative interpretation then, is that there is simply a trend for the more IR-luminous galaxies to be powered by AGN and the less IR-luminous ones to be powered by starbursts. We will not be able to decide for certain between these two interpretations until the long-slit data are analyzed in detail for all of the sample galaxies. A preliminary analysis indicates that the situation in M82 (progressively more "LINER-like" line ratios at larger radii) is indeed common in our sample galaxies.

It is also quite possible that the nature of the principal ionization source could change as the galaxy evolves. For example, a nuclear starburst might be a precursor of more energetic types of activity, such as a Seyfert nucleus, or a quasar (cf. Norman and Scoville 1988; Sanders et al. 1988;

\footnotetext{
${ }^{3}$ For the ICC FIRG galaxies with multiple nuclei, the $L_{\text {FIR }}$ of the entire system was divided equally into each nucleus to arrive at the averages listed above. A high percentage of the system $L_{\text {FIR }}$ may, however, come from only one nucleus, but the true distribution of the far-infrared luminosity is uncertain due to the poor resolution available from $I R A S$.
} 
Heckman, Armus, and Miley 1989). One way of exploring this possibility is to determine whether the emission-line properties of the IR color-selected FIRGs are related to the age of the dominant stellar population. We can do this by comparing the absorption/emission-line spectra and the continuua of the AGN-like and $\mathrm{H}$ II-like groups of ICSS FIRGs.

It was noted in the previous section that the 53 IR colorselected nuclei have unusually low values for $E_{\mathrm{Mg}}$, and for the galaxies with high signal-to-noise ratios well off the nucleus at least, the value of $E Q W_{M g}$ stays weak at radii up to $4 \mathrm{kpc}$. The galaxies with nuclear emission-line flux ratios similar to those found in AGNs tend to have larger $\mathrm{EQW}_{\mathrm{Mg}}$ than do the galaxies with nuclear spectra resembling $\mathrm{H}$ II regions (average $\mathrm{EQW}_{\mathrm{Mg}} \sim 1.1 \AA$ vs. $0.5 \AA$ respectively). This can be seen in Figure 15, and it implies that the characteristic age of the stellar population in the AGN-type nuclei is older than in the $\mathrm{H}$ II region-type nuclei. Likewise, the distributions of $\mathrm{EQW}_{\mathrm{H} \alpha}$ are different for the two classes of ICSS FIRGs. As can be seen in Figure 16, the $\mathrm{H}$ II-like galaxies have larger $\mathrm{EQW}_{\mathrm{H} \alpha}$, on average, than the AGN-like galaxies (median log $\mathrm{EQW}_{\mathrm{H} \alpha} \sim$ 2.0 vs. $\sim 1.5$, respectively). The nuclei classified as $\mathrm{AGN}$-like could thus be interpreted as containing a relatively larger population of older $\left(>10^{7} \mathrm{yr}\right)$ stars than the sample galaxies classified as $\mathrm{H}$ II-like. The galaxies that appear to harbor active nuclei might therefore be at a more advanced stage in their development, but an overall evolutionary link cannot at this point be established among our IR color-selected galaxies. For example, the difference between the two classes may primarily
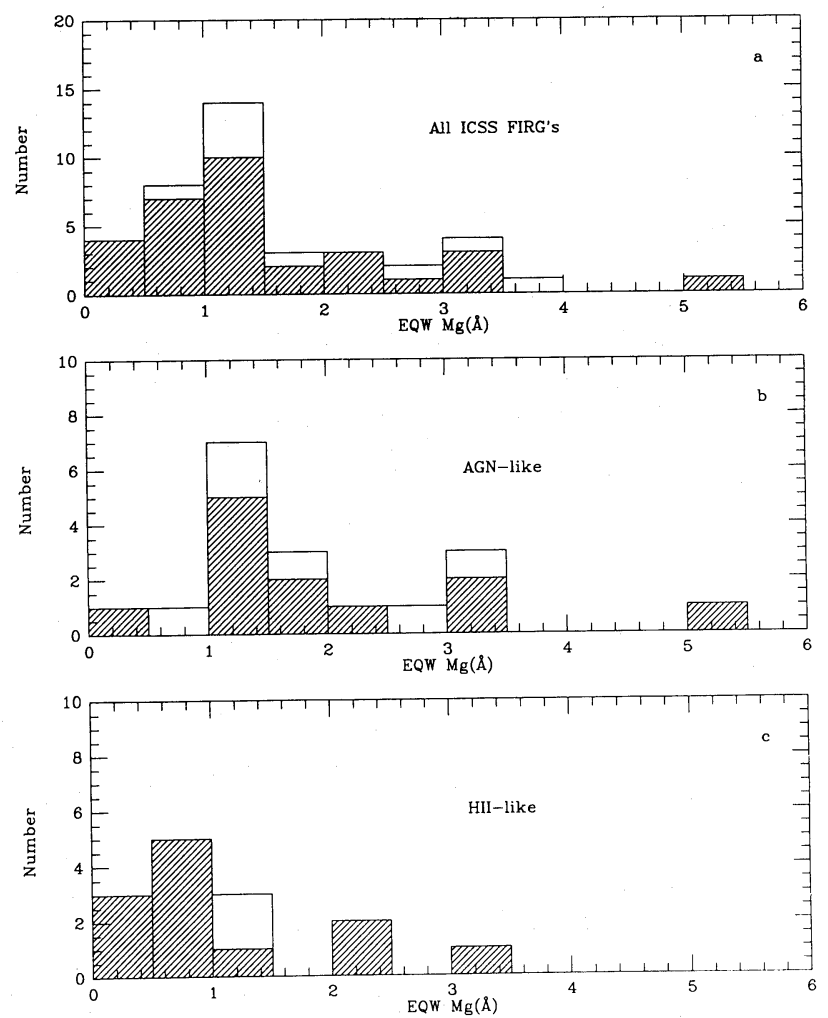

FIG. 15.-(a) Distribution of the equivalent width of the $\mathrm{Mg} \mathrm{I}$ absorption line for all of the ICSS FIRGs, $(b)$ for the ICSS FIRGs with nuclear spectra resembling AGN (LINERs), and (c) for the ICSS FIRGs with nuclear spectra resembling $\mathrm{H}$ II regions. Shaded areas denote upper limits to the $\mathrm{Mg}$ I equivalent width.
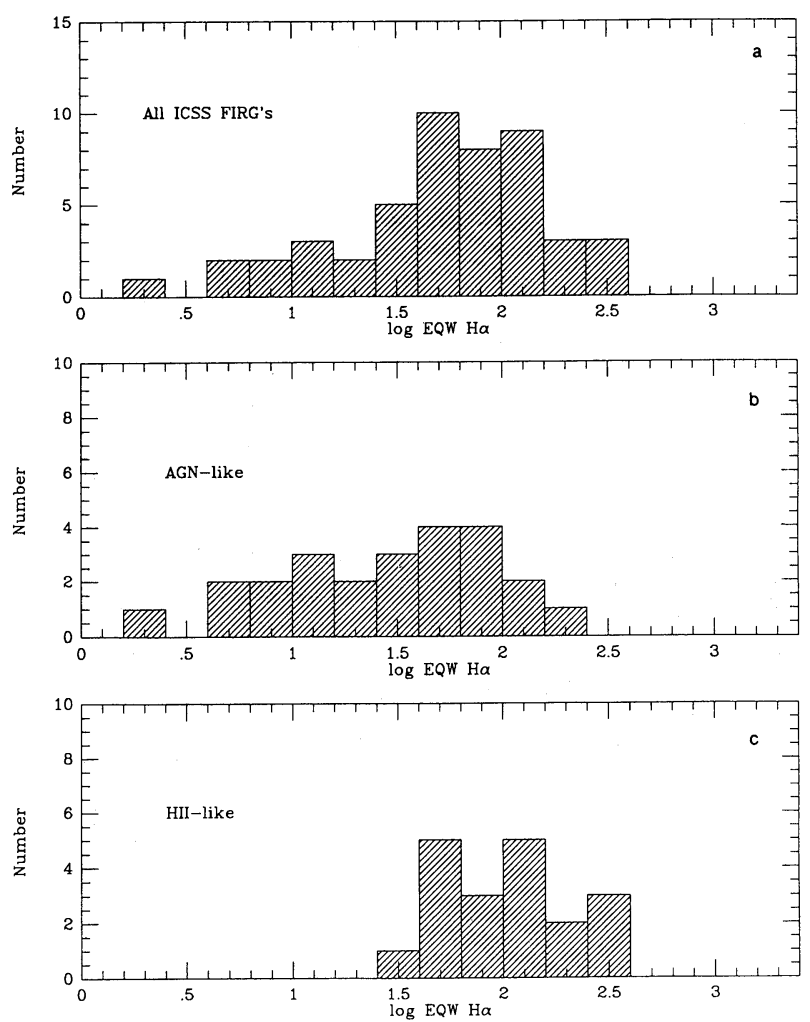

Fig. 16. (a) Distribution of the equivalent width of the $\mathrm{H} \alpha$ emission line for all the ICSS FIRGs, $(b)$ for the ICSS FIRGs with nuclear spectra resembling AGN (LINERs), and (c) for the ICSS FIRGs with nuclear spectra resembling $\mathrm{H}$ II regions.

reflect the larger effective spectroscopic aperture used for the more distant AGN-like FIRGs (see above).

The simplest explanation for the diverse nuclear properties seen in our sample galaxies, is that these objects do not make up a distinct class of their own. It is quite possible that we have managed to glimpse these galaxies at a time when they have nothing more in common than the shape of their infrared continua (i.e., they have only their characteristic dust temperatures in common). Indeed, we may be looking at an assortment of galaxies whose optical nuclear spectra would otherwise simply cause them to be placed into what are now the accepted categories of galactic activity (primarily starburst or LINER). Whatever the cause, these galaxies have now been "turned on" and are all in the process of heating up their interstellar material. However, if our sample is really a mixture of starbursts and $A G N$, it is perplexing in that objects which are unambiguously AGN (Seyferts or quasars) are so rare in our sample. Moreover, the fact that these IR color-selected galaxies do seem to have many common properties (as outlined above) leads us to the conclusion that perhaps throughout the sample there is a single, dominant driving force behind most of what we observe, and this driving force is an enhanced episode of star formation.

\section{v. CONCLUSIONS}

Optical spectra of the nuclear regions (kpc scale) of 47 farinfrared galaxies (FIRGs) chosen to have far-infrared spectral energy distributions similar to those of M82, Arp 220, NGC 6240 , and NGC 3690, have been presented and discussed. These data bear directly on the fundamental nature of FIRGs and their relationship to other types of active galaxies. 
In the most popular model for the FIRGs the far-infrared emission is powered by star formation with UV radiation from young stars reprocessed by dust. Our data represent direct evidence for the two main ingredients of this model.

\section{a) Young Stars}

1. Emission-line flux ratios of $\sim 40 \%$ of the galaxies indicate that photoionization by hot stars dominates the heating of the gas. Most of the other galaxies have LINER-like spectra, or are intermediate between LINERs and $\mathrm{H}$ II regions.

2. The weakness of the $\mathrm{Mg}$ I $b$ stellar absorption line (and of other indicators of old stellar populations) implies that a young stellar population or an AGN dominates the visible spectrum in all of the galaxies.

3. The simultaneous presence of weak $\mathrm{Mg}$ I absorption and moderately strong $\mathrm{TiO}$ absorption at $\lambda \sim 6250 \AA$ is evidence that a population with an age $\sim 10^{7} \mathrm{yr}$ (red supergiants) is making a significant contribution to the red light.

4. The fact that the regions with weak $\mathrm{Mg}$ I absorption lines are spatially extended (1-4 kpc) argues for young stars and not an AGN.

5. The continuum colors of a majority of the sample galaxies (when dereddened using the Balmer decrement) are also consistent with those of a young population of stars. The use of the Balmer decrement is suggested by its good correlation with color and with the equivalent width of interstellar $\mathrm{Na}$ I $\mathrm{D}$ absorption lines.

6. The extinction-corrected luminosity of the optical continuum is consistent with young stars powering typically at least $25 \%$ of the far-infrared emission.

7. Strong Balmer absorption lines suggest that a large intermediate-age stellar population is present in some cases.

8. Balmer emission-line equivalent widths are intermediate between those found in nuclear and disk $\mathrm{H}$ II regions. They may reflect a mixture of a large population of young $\left(<10^{7} \mathrm{yr}\right.$ old) and intermediate $\left(10^{8}-10^{9}\right.$ yr old) age stars.

9. The presence of Wolf-Rayet features in at least one and probably five galaxies is additional evidence for a significant population of young, massive stars.

\section{b) Dust and Cool Gas}

1. Balmer decrements imply that several magnitudes of extinction at $\mathrm{H} \beta$ are typically present. This is consistent with the $\mathrm{H}$ I column densities as measured by Mirabel and Sanders (1988), assuming a normal gas-to-dust ratio.

2. Large $\mathrm{Na}$ I $\mathrm{D}$ equivalent widths indicative of high interstellar column densities of $\mathrm{Na}$ I are present. These $\mathrm{Na}$ I $\mathrm{D}$ equivalent widths correlate well with $H \alpha / \mathbf{H} \beta$, and with the color of the continuum. The equivalent width of the $\mathrm{Na}$ I $\mathrm{D}$ multiplet does not correlate with the strength of lines produced by cool stars (e.g., the Mg I $\lambda 5174 b$ band).

3. The continuum colors are usually very red, despite the clear spectroscopic signatures of a young stellar population. These red colors correlate well with $\mathrm{H} \alpha / \mathrm{H} \beta$ and with the equivalent width of the $\mathrm{Na}$ I $\mathrm{D}$ lines.

Large populations of young stars, then, appear to be present in all of our infrared color-selected FIRGs, but their contribution to the overall energy budget of these galaxies is in doubt. We have shown that these stars may power $25 \%$ or more of the far-infrared emission from our sample of FIRGs. However, the emission-line flux ratios, and line widths of many of the galaxies suggest an alternate, or additional source of energy is present as well. While this energy source could be an AGN, it is possible that the AGN-like (LINER) emission-line flux ratios, large line widths, and blue asymmetric emission-line profiles reflect outflowing material that is shock-heated by a starburstinduced, supernovae-driven "superwind" (see McCarthy, Heckman, and van Breugel 1987; Heckman, Armus, and Miley 1987, 1989). This possibility is being tested by a detailed investigation of the long-slit data. Thus far, it is suggestive that we see very few unambiguous AGN (e.g., Seyferts or QSO's) in our sample. We believe this favors the "superwind" rather than the "AGN" interpretation.

An important question, therefore, is whether our IR colorselected FIRGs represent a single, unique phenomenon, or several different ones united only by the temperature of their far-infrared-emitting dust. An evolutionary sequence among our FIRGs may be indicated by observational evidence for a more dominant young stellar population in the galaxies with nuclear spectra resembling $\mathrm{H}$ II regions, compared to those with nuclear spectra similar to AGN (LINERs). The evidence concerning these points is however, inconclusive, and the establishment of a link between our far-infrared color-selected galaxies and the more classical forms of galactic activity must wait for further analysis of our long-slit spectroscopic data.

We would like to thank the staff at NOAO, and CTIO for their help in obtaining the data reported in this paper. We would also like to thank P. S. Conti for a valuable discussion on Wolf-Rayet stars. T. H. and L. A. are supported by the NSF under grant AST 85-15896 and by the NASA SADAP/IRAS program. This paper is the result of ongoing research toward fulfillment of the requirements of the Ph.D. degree at the University of Maryland.

\section{REFERENCES}

Armus, L., Heckman, T. M., and Miley, G. K. 1988, Ap. J. (Letters), 326, L45.

Baldwin, J. A., Phillips, M. M., and Terlevich, R. 1981, Pub. A.S.P., 93, 5.

Balzano, V. A. 1983, Ap. J., 268, 602.

Bica, E., Alloin, D., and Schmidt, A. 1989, preprint

Binette, L., Dopita, M. A., and Tuohy, I. R. 1985, Ap. J., 297, 476.

Bland, J., and Tully, R. B. 1988, preprint.

Canto, J., Elliott, K. H., Meaburn, J., and Theokas, A. C. 1980, M.N.R.A.S., 193, 911.

Cataloged Galaxies and Quasars Observed in the IRAS Survey. 1985, prepared

by C. J. Lonsdale, G. Helou, J. C. Good, and W. Rice (Pasadena: JPL).

Chevalier, R. A., and Clegg, A. W. 1985, Nature, 317, 44.

Cutri, R. M., Rieke, G. H., and Leofsky, M. J. 1984, Ap. J., 287, 566.

Dahari, O., and DeRobertis, M. M. 1988, Ap. J. Suppl., 67, 249.

DeGioia-Eastwood, K. 1985, Ap. J., 288, 175.

Feigelson, E. D., and Nelson, P. I. 1985, Ap. J., 293, 192.

Ferland, G. J., and Netzer, H. 1983, Ap. J., 264, 105.

Fosbury, R.A. E., and Wall, J. V. 1979, M.N.R.A.S., 189, 79.

Gehrz, R. D., Sramek, R. A., and Weedman, D. W. 1983, Ap. J., 267, 551.
Heckman, T. M., Armus, L., and Miley, G. K. 1987, A.J., 93, 276. . 1989, preprint.

Heckman, T. M., Balick, B., and Crane, P. C. 1980, Astr. Ap. Suppl., 40, 295.

Jacoby, G. H., Hunter, D. A., and Christian, C. A. 1984, Ap. J. Suppl., 56, 257. Kennicutt, R. C. 1983, Ap. J., 272, 54.

Kennicutt, R. C., Keel, W. C., and Blaha, C. A. 1989, A.J., 97, 1022.

Kraemer, S. B. 1985, Ph.D. thesis, University of Maryland.

Kurucz, R. L. 1979, Ap. J. Suppl., 40, 1.

McCall, M. L., Rybski, P. M., and Shields, G. A. 1985, Ap. J. Suppl., 57, 1.

McCarthy, P. J., Heckman, T. M., and van Breugel, W. 1987, A.J., 93, 264.

Miller, G.E., and Scalo, J. M. 1979, Ap. J. Suppl., 41, 513.

Mirabel, I. F., and Sanders, D. B. 1988, Ap. J., 335, 104.

Norman, C., and Scoville, N. Z. 1988, Ap. J., 332, 124.

O'Connell, R. W. 1973, A.J., 78, 1074.

Osterbrock, D. E. 1988, Astrophysics of Gaseous Nebulae and Active Galactic Nuclei (Mill Valley: University Science Books).

Rice, W., Lonsdale, C. J., Soifer, B. T., Neugebauer, G., Kopan, E. L., Lloyd, L. A., DeJong, T., and Habing, H. J. 1988, Ap. J. Suppl., 68, 91. 
Rieke, G. H., Cutri, R. M., Black, J. H., Kailey, W. F., McAlary, C. W., Lebofsky, M. J., and Elston, R. 1985, Ap. J., 290, 116.

Rieke, G. H., Lebofsky, M. J., Thompson, R. I., Low, F. J., and Tokunaga, A. T. 1980, Ap. J., 238, 24.

Sanders, D. B., Soifer, B. J., Elias, J. H., Madore, B. F., Matthews, K., Neugebauer, G., and Scoville, N. Z. 1988, Ap. J., 325, 74

Shull, J. M., and McKee, C. F. 1979, Ap. J., 227, 131

Soifer, B. T., et al. 1984, Ap. J. (Letters), 278, L71.
Stauffer, J. R. 1982, Ap. J.Suppl., 50, 517.

Stein, W. A., and Soifer, B. T. 1983, Ann. Rev. Astr. Ap., 21, 177.

Strömgren, B. 1948, Ap. J., 108, 242.

Veilleux, S., and Osterbrock, D. E. 1987, Ap. J. Suppl., 63, 295.

Whittle, M. 1985, M.N.R.A.S., 213, 1 .

Wilson, A. S., and Heckman, T. M. 1984, Astrophysics of Active Galaxies and Quasi-Stellar Objects (Mill Valley: University Science Books). Wyse, R. F. G. 1985, Ap. J., 299, 593.

Note added in proof.-As an additional check on the fraction of $L_{\mathrm{BOL}}$ we are seeing in the optical, Ken Chambers has graciously run a Bruzual starburst model with a Salpeter IMF and a time constant of $t=10^{7} \mathrm{yr}$. In this model $L_{48} / L_{\mathrm{BOL}}$ ranges from $\sim 5 \%$ at $t \leq 10^{7}$ yr up to $\sim 20 \%$ at $t \sim 10^{8}$ yr. For our sample galaxies we measure an average $L_{48} / L_{\mathrm{BOL}} \sim 5 \%$. Thus, the young stars that we see in the optical (after correcting for extinction) may power a significant fraction $(\geq 25 \%)$ of the total luminosity of the ICSS FIRGs.

LeE ARMUS: Astronomy Program, University of Maryland, Space Sciences Building, College Park, MD 20742

Timothy M. Heckman: Department of Physics and Astronomy, The Johns Hopkins University, Baltimore, MD 21218

GeORGE K. MiLeY: Leiden Observatory, Niels Bohrweg 2, 2300 RA Leiden, The Netherlands 